\title{
Cdk5 is essential for synaptic vesicle endocytosis
}

\author{
Timothy C. $\operatorname{Tan}^{1}$, Valentina A. Valova ${ }^{1}$, Chandra S. Malladi ${ }^{1}$, Mark E. Graham ${ }^{1}$, Leise A. Berven ${ }^{1}$, Orla J. Jupp ${ }^{6}$, \\ Gurdip Hansra $^{1}$, Sonya J. McClure ${ }^{2}$, Boris Sarcevic ${ }^{3}$, Ross A. Boadle ${ }^{4}$, Martin R. Larsen ${ }^{5}$, Michael A. Cousin ${ }^{6}$ \\ and Phillip J. Robinson ${ }^{1,7}$
}

\begin{abstract}
Synaptic vesicle endocytosis (SVE) is triggered by calcineurin-mediated dephosphorylation of the dephosphin proteins. SVE is maintained by the subsequent rephosphorylation of the dephosphins by unidentified protein kinases. Here, we show that cyclindependent kinase 5 (Cdk5) phosphorylates dynamin I on Ser 774 and Ser 778 in vitro, which are identical to its endogenous phosphorylation sites in vivo. Cdk5 antagonists and expression of dominant-negative Cdk5 block phosphorylation of dynamin I, but not of amphiphysin or AP180, in nerve terminals and inhibit SVE. Thus Cdk5 has an essential role in SVE and is the first dephosphin kinase identified in nerve terminals.
\end{abstract}

SVE is required for maintaining the small pool of synaptic vesicles within nerve terminals after exocytosis and thus is important for sustaining synaptic transmission. It is activated by a calcineurin-mediated dephosphorylation event that is stimulated by depolarization-dependent calcium influx ${ }^{1-4}$. Eight proteins, collectively called dephosphins, are dephosphorylated by calcineurin in nerve terminals. They are dynamin I, synaptojanin, amphiphysins I/II, epsin, eps15, AP180 and PIP kinase $I \gamma^{5,6}$. Each member of this structurally unrelated group of proteins is implicated in SVE at different stages ${ }^{5,6}$.

The dephosphins are constitutively phosphorylated in resting nerve terminals, and their rephosphorylation after termination of SVE is essential for subsequent rounds of endocytosis. The protein kinases that mediate the essential rephosphorylation step are still undetermined. Protein kinase C (PKC) may be a dephosphin kinase, as PKC antagonists such as Ro31-8220 inhibit repetitive rounds, but not the initial round, of $\mathrm{SVE}^{3}$. PKC antagonists also inhibit the rephosphorylation of at least two dephosphins in nerve terminals, dynamin I and synaptojanin ${ }^{3}$, but not amphiphysin or AP180, implicating the phosphorylation status of the former proteins as essential for SVE. PKC phosphorylates dynamin I in vitro on Ser 795 and prevents the interaction of dynamin I with membrane phospholipids in vitro ${ }^{7}$. Thus, PKC may be the dephosphin kinase whose activity is essential for maintenance of SVE.

Dynamin I is a GTPase whose activity is essential for synaptic vesicle fission $^{8}$. It has a carboxy-terminal proline-rich domain (PRD) containing numerous binding motifs for src-3-homology ( $\mathrm{SH} 3$ ) domains. The interaction of the dynamin I PRD with SH3-domain-containing proteins such as amphiphysin I/II and endophilin is essential for $\mathrm{SVE}^{9,10}$. The PRD is also the site of endogenous dynamin I phosphorylation ${ }^{11,12}$ and phosphorylation by other protein kinases in vitro, including PKC (Ser 795) ${ }^{7}$, casein kinase II (ref. 12), cdc2 (ref. 13), Erk/MAPK ${ }^{14}$ and minibrain kinase ${ }^{15}$. The endogenous dynamin I kinase in nerve terminals remains to be identified.
Cdk5 is a candidate, as the PRD contains multiple Ser-Pro or ThrPro motifs that are targets for cyclin-dependent kinases ${ }^{16}$. In the adult brain, Cdk5 and its activator protein $\mathrm{p} 35$ are enriched in nerve terminals, with p35 localized to vesicular fractions ${ }^{17}$ suggesting a role for Cdk5 in synaptic vesicle recycling. A growing list of in vitro Cdk5 substrates are nerve terminal proteins with essential roles in synaptic vesicle recycling, including synapsin I (ref. 18), munc-18 (ref. 19) and amphiphysin I (ref. 20). In this study, we wanted to determine whether Cdk5 is the dynamin I kinase in adult nerve terminals and if Cdk5dependent phosphorylation is required for maintenance of SVE. We present evidence that Cdk5 phosphorylates dynamin I in vitro on the same phosphorylation sites as the endogenous dynamin I kinase, ruling out PKC as the endogenous kinase. Furthermore, inhibition of Cdk5 blocked both rephosphorylation of dynamin I and repetitive rounds of SVE in nerve terminals. Our results demonstrate that Cdk5 activity is essential for SVE and represents the first identified dephosphin kinase.

\section{RESULTS}

Cdk5 phosphorylates dynamin I in vitro

To determine whether dynamin I is a Cdk5 substrate in vitro, we phosphorylated purified dynamin I with recombinant Cdk5 and its activator p25. Both Cdk5 and recombinant PKC- $\alpha$ phosphorylated dynamin I to a similar extent (Fig. 1a) at its C-terminal tail (Fig. 1b). Endogenous dynamin I is also phosphorylated at its C-terminal tail, predominantly on serine residues ${ }^{12}$. The same pattern of phosphorylation was observed for both Cdk5- and PKC- $\alpha$-mediated phosphorylation of dynamin I in vitro (Fig. 1c). Cdk5 also phosphorylated a glutathione $S$-transferase (GST) fusion protein containing the entire dynamin I PRD (GST-PRD; Fig. 1d), which contains multiple copies of motifs required by the kinase ${ }^{16}$ (Fig. 1e). PKC- $\alpha$ did not phosphorylate GST-PRD, suggesting that PKC- $\alpha$ can only phosphorylate full-length

${ }^{1}$ Cell Signalling Unit, Children's Medical Research Institute, Locked Bag 23, Wentworthville, NSW 2145, Australia. ${ }^{2}$ Royal Hospital for Women in Randwick, Barker St, Randwick, NSW 2031, Australia. ${ }^{3}$ Cancer Research Program, Garvan Institute of Medical Research, 384 Victoria Street, Darlinghurst, NSW 2010 , Australia. ${ }^{4}$ Electron Microscope Laboratory, Institute of Clinical Pathology and Medical Research, Westmead Hospital, Westmead, NSW, 2145 , Australia. ${ }^{5}$ Australian Proteomic Analysis Facility, Macquarie University, Balaclava Road, North Ryde, NSW 2109, Australia. ${ }^{6}$ Membrane Biology Group, Division of Biomedical Sciences, George Square, University of Edinburgh, EH8 9XD, Scotland, UK. ${ }^{7}$ Correspondence should be addressed to P.J.R. (e-mail: phrobins@mail.usyd.edu.au.). 


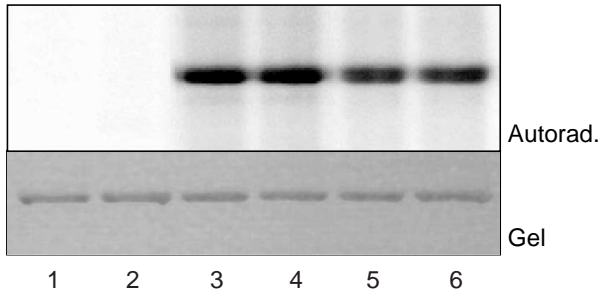

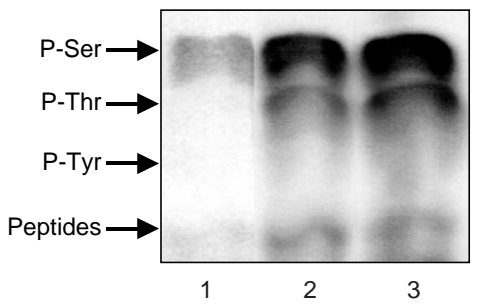

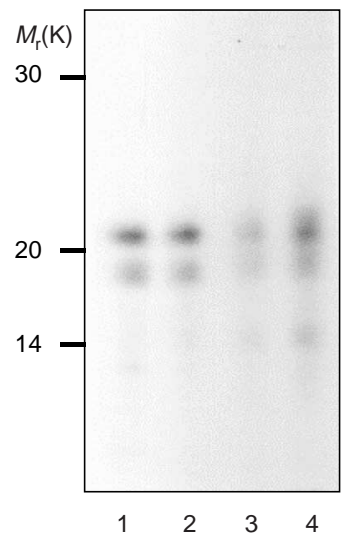

Figure 1 Dynamin I is an in vitro substrate for Cdk5. (a) Phosphorylation of purified dynamin I alone (lanes 1 and 2) or with Cdk5 (lanes 3 and 4) or PKC- $\alpha$ (lanes 5 and 6) in vitro. (b) V8 protease digestion of dynamin I phosphorylated by Cdk5 yields two phosphopeptides at 21 and $19 \mathrm{~K}$ (lanes 1 and 2). PKC phosphorylates the same peptides (lanes 3 and 4) derived from the C-terminal tail of dynamin la and Ib splice variants ${ }^{12}$. (c) Phosphoamino acid analysis shows that Cdk5 (lane 2) and PKC (lane 3)

dynamin I. This hypothesis is consistent with previous data, where synthetic peptides encompassing the PKC phosphorylation site were not phosphorylated ${ }^{7}$. Thus, dynamin I is a Cdk5 substrate in vitro.

Phosphorylation by Cdk5 reduced its ability to associate with phospholipids (Fig. 2a). This supports the hypothesis that phosphodynamin is predominantly cytosolic in nerve terminals and suggests that dynamin dephosphorylation may contribute to its recruitment to sites of SVE at the plasma membrane. Phosphorylation by Cdk5 also increased the GTPase activity of dynamin (Fig. 2b), whereas phosphorylation by PKC did not. Phosphorylation by PKC is known to stimulate GTPase activity ${ }^{12}$, and the reason for the discrepancy with the current results relates to methodological differences (see Supplementary Information, Fig. S1).

The PRD of dynamin I is essential for SVE, as disruption of its interactions with the SH3 domains of amphiphysin I and endophilin inhibits the process ${ }^{9,10,21}$. Next, we examined whether in vitro phosphorylation of the dynamin I PRD by Cdk5 could regulate its interaction with the SH3 domain of amphiphysin I. Phosphorylation of dynamin I by Cdk5 in vitro did not affect binding of the amphiphysin I or Grb2 SH3 domains (data not shown). However, phosphorylation of dynamin I was blocked when it was pre-incubated with the amphiphysin I SH3 domain before addition of Cdk5 (Fig. 2c). Cdk5-mediated phosphorylation of dynamin I was not affected by a similar incubation with the SH3 domain of Grb2 (Fig. 2d), despite Grb2 having an overlapping binding site with amphiphysin I (ref. 22; Fig. 1e). Phosphorylation of dynamin I by PKC- $\alpha$ was not affected by the amphiphysin I SH3 domain (Fig. 2c). Thus, Cdk5-mediated phosphorylation of dynamin I d
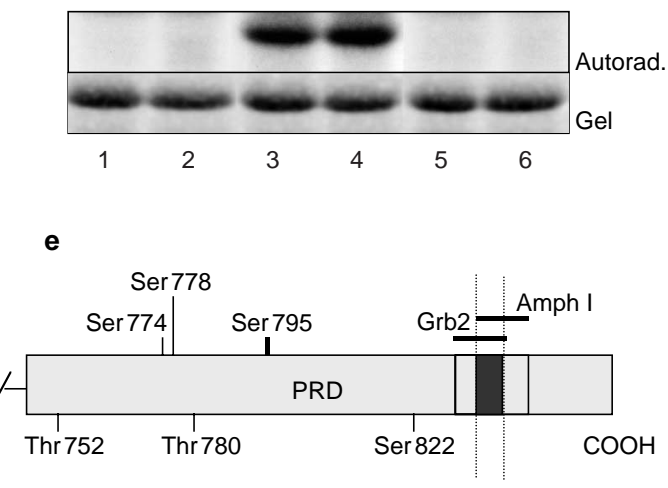

phosphorylate dynamin I predominantly on serine residues, as also observed from endogenous dynamin I purified from ${ }^{32} \mathrm{P}$-labelled intact synaptosomes (lane 1). (d) Phosphorylation of GST-PRD alone (lanes 1 and 2), with Cdk5 (lanes 3 and 4 ) or PKC- $\alpha$ (lanes 5 and 6) in vitro. (e) Potential phosphorylation sites for Cdk5 and the PKC phosphorylation site (Ser 795) in the PRD of dynamin I. Binding sites for the SH3 domains of Grb2 and amphiphysin I are indicated. All results are shown in duplicate, except for $\mathbf{c}$.

is regulated by its interaction with amphiphysin I, suggesting that phosphorylation of dynamin I might only occur after release of amphiphysin I, possibly at the termination of SVE.

We used matrix-assisted laser desorption/ionization-time-of-flight mass spectrometry (MALDI-TOF MS) to reveal the in vitro phosphorylation sites on dynamin I for Cdk5. Most of the signals detected in isolated tryptic phosphopeptides corresponded to sequences from dynamin I containing at least two acidic residues (Fig. 3a). Two signals of average relative molecular mass $1,138.53$ and 1,293.77 corresponded to the masses of the two mono-phosphopeptides, SPTSSPTPQR (Dyn I 774-783) and RSPTSSPTPQR (Dyn I 773-783). Dephosphorylation of these peptides with alkaline phosphatase produced a selective loss of 80 and resulted in two new signals at $1,057.49$ and 1,213.34, corresponding to the unphosphorylated peptides (Fig. 3b). No peptide containing Ser 795 was detected, indicating that Cdk5 phosphorylates site(s) distinct from $\mathrm{PKC}^{7}$. To determine at which sites dynamin I was phosphorylated in vivo, the phosphorylation sites on endogenous dynamin I were identified. The peptide mass maps from endogenous dynamin I closely resembled the pattern observed with in vitro phosphorylation using Cdk5 (Fig. 3c, d). This suggests that dynamin I is not phosphorylated by PKC in nerve terminals and is consistent with Cdk 5 being the endogenous dynamin I kinase.

\section{Dynamin I is phosphorylated by Cdk5 in nerve terminals}

As dynamin I was mono-phosphorylated on the peptide SPTSSPTPQR by both Cdk5 in vitro and by its endogenous kinase in vivo, we determined which residue was the primary phosphate acceptor site. Ser 774 
a

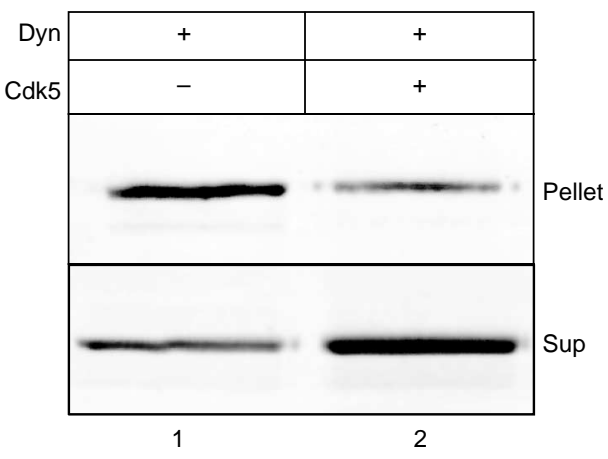

C

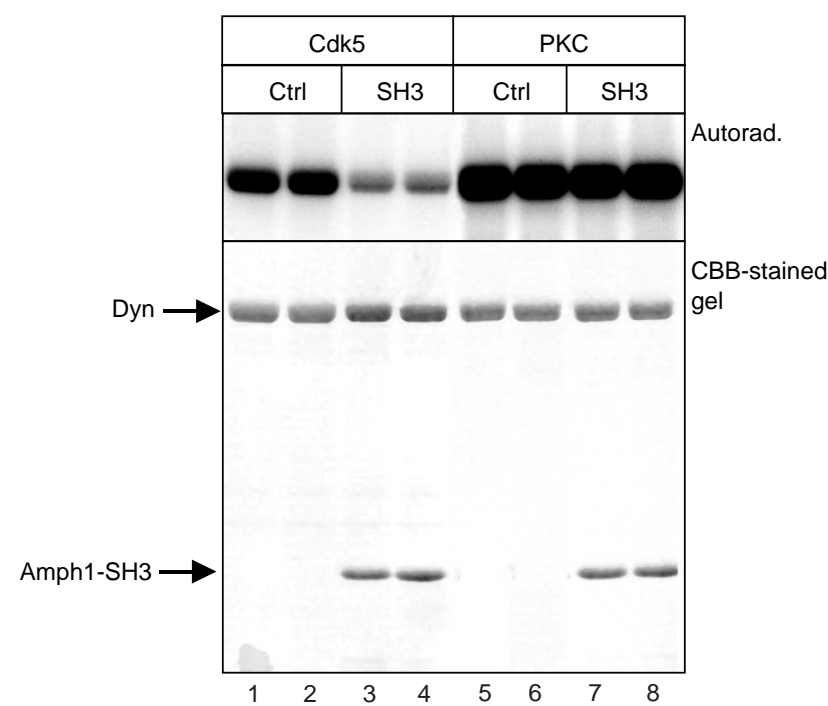

b

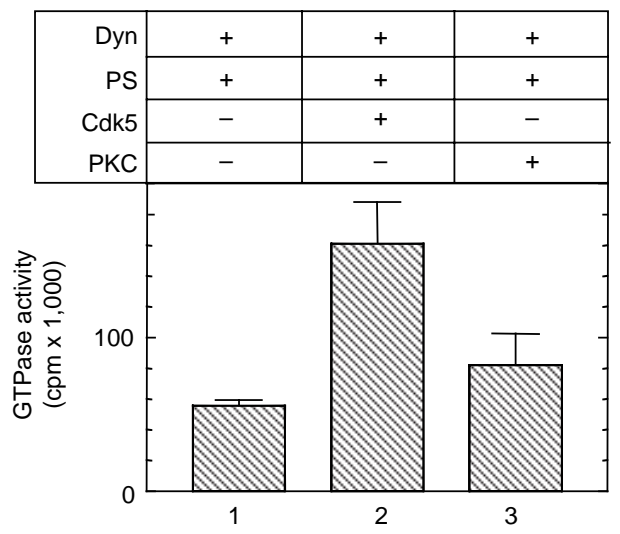

d

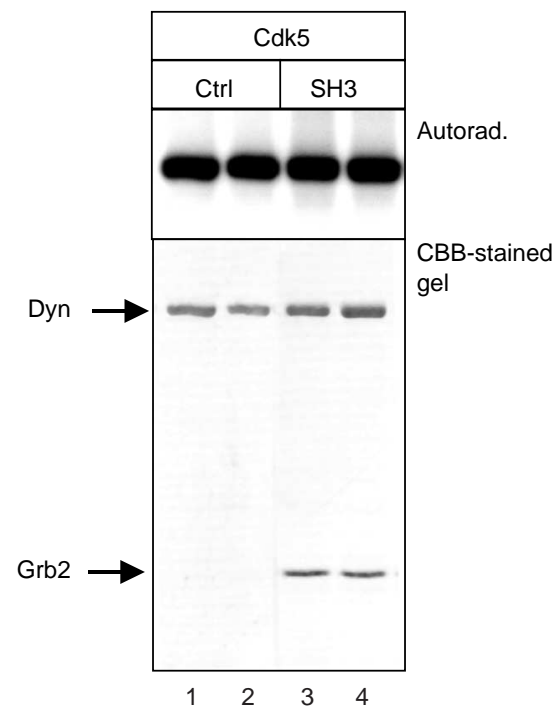

Figure 2 In vitro functional effects of Cdk5 phosphorylation of dynamin I. (a) Phosphorylation of dynamin reduces its ability to bind phospholipids. Dynamin was phosphorylated for 60 min with Cdk5 or mock-phosphorylated in its absence then incubated with phospholipid-coated beads. Bound and free dynamin were separated by centrifugation ${ }^{7}(n=3)$. (b) The effect of Cdk5 phosphorylation on dynamin's GTPase activity. Dynamin I ( $5 \mu \mathrm{g})$ was phosphorylated with Cdk5/p25 or PKC- $\alpha$ in the presence of Mg/ATP ( $1 \mathrm{mM}$ ), phosphatidylserine (PS, $10 \mu \mathrm{g} \mathrm{ml}^{-1}$ ) and $200 \mu \mathrm{M}$ calcium for $1 \mathrm{~h}$ at $30{ }^{\circ} \mathrm{C}$ before stopping the reaction with sodium chloride (see Supplementary
Information Methods). Phosphorylated dynamin I was then assayed for GTPase activity ( $n=3$, see Supplementary Information Methods). (c) Dynamin I was incubated with (SH3) or without (control) recombinant amphiphysin I-SH3 domain and then phosphorylated by Cdk5 or PKC, as indicated. (d) Dynamin I was incubated with (SH3) or without (control) recombinant Grb2-SH3 domain and then phosphorylated by Cdk5.

Experiments in $\mathbf{c}$ and $\mathbf{d}$ were performed in duplicate and indicate either dynamin I phosphorylation (autorad) or total dynamin I and SH3 domain present (Coomassie Brilliant Blue (CBB)-stained gel). and Ser 778 both reside in an appropriate context predicted for Cdk5 substrates, being followed by a Pro residue ${ }^{16}$. Ser 777 was not followed by a Pro residue and was not considered further. Phospho-specific antibodies were raised against phospho-Ser 774 and phospho-Ser 778 to determine which was the primary phosphorylation site. Both antibodies recognized dynamin I phosphorylated in vitro by Cdk5, but not by PKC- $\alpha$ (Fig. 3e). Conversely, antibodies to phospho-Ser 795 recognized dynamin I after in vitro phosphorylation by PKC- $\alpha$, but not Cdk5 (Fig. 3e). Thus, Cdk5 can phosphorylate dynamin I on either Ser 774 or Ser 778 in vitro. Phosphorylation of Thr 780 cannot be excluded, but was not investigated further (Fig. 1c).

To determine whether Cdk5 phosphorylated dynamin I in intact nerve terminals, we used a well-characterized Cdk5 antagonist, roscovitine $^{23}$. First, we tested the specificity of roscovitine for Cdk5 relative to
PKC, as PKC is proposed to be the dynamin I kinase in nerve terminals. Roscovitine inhibited in vitro dynamin I phosphorylation by Cdk5, but not by PKC- $\alpha$, in a concentration-dependent manner (see Supplementary Information, Fig. S2a). To confirm roscovitine effectively discriminates Cdk5 from PKC activities, we used a second assay based on a synthetic peptide substrate. PKC- $\alpha$ activity was again unaffected by roscovitine over a broad concentration range (see Supplementary Information, Fig. S2b). The relatively selective PKC inhibitor Ro31-8220 inhibits dynamin I phosphorylation in intact nerve terminals ${ }^{3}$, originally suggesting that PKC may be the endogenous dynamin I kinase. Ro31-8220 also inhibits other protein kinases ${ }^{24}$ and we found it inhibited phosphorylation of dynamin I by PKC- $\alpha$ in vitro, but was even more potent for Cdk5 (see Supplementary Information, Fig. S2a). Thus, the inhibition of dynamin I phosphorylation in nerve 
a
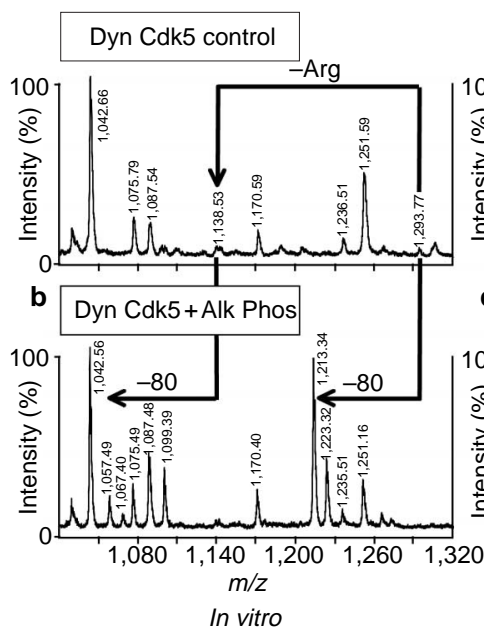

C



e

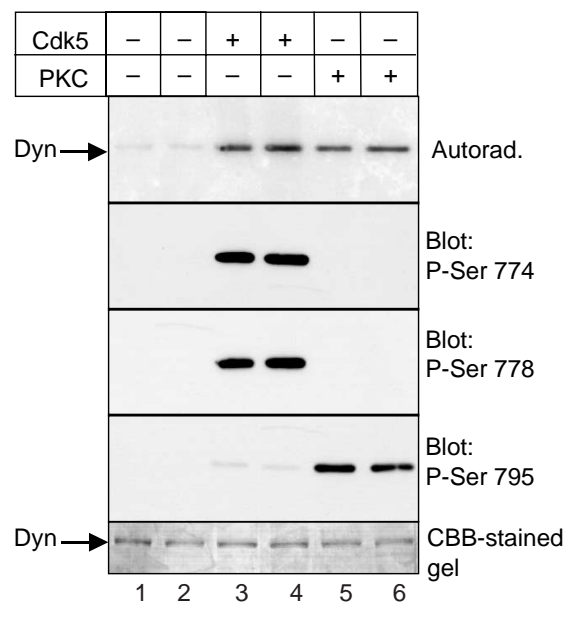

Figure 3 Dynamin phosphorylation sites in vitro and in vivo. (a) MALDI-TOF MS peptide mass map of tryptic fragments isolated by $\mathrm{Fe}^{3+}-$ IMAC columns from dynamin I phosphorylated in vitro by Cdk5. Two of the signals corresponding to phospho-SPTSSPTPQR $(\mathrm{m} / z 1,138.53)$ and phosphoRSPTSSPTPQR $(m / z 1,293.77)$ are cleavage variants derived from the same sequence. (b) MALDI-TOF MS peptide mass map of the same sample after treatment with alkaline phosphatase. There are two new signals $(\mathrm{m} / \mathrm{z}$ $1,057.49$ and $1,213.34$ ) corresponding to the non-phosphorylated peptides SPTSSPTPQR and RSPTSSPTPQR. (c) Similar analysis on endogenous dynamin I isolated from intact synaptosomes shows essentially identical results. (d) After alkaline phosphatase treatment, two new signals appear $(\mathrm{m} / \mathrm{z}$ 1,057.33 and 1,213.15), corresponding to a mass reduction of 80 . (e) Antibodies to peptides containing phospho-Ser 774 and phospho-Ser 778 detect purified dynamin I phosphorylated in vitro by recombinant Cdk5 (lanes 3 and 4), but not PKC (lanes 5 and 6). Conversely antibodies to phospho-Ser 795 detect dynamin phosphorylated in vitro by PKC, but not Cdk5. Phosphorylated dynamin I is shown at the top (Autorad.), whereas total dynamin I levels are shown at the bottom (CBB-stained gel). terminals by Ro31-8220 (ref. 3) may have been a result of its inhibition of Cdk5, rather than PKC.

Dynamin I is constitutively phosphorylated in nerve terminals and dephosphorylated by calcineurin when SVE is stimulated by depolarization-dependent calcium influx $(\mathrm{S} 1)^{5}$. After removal of the stimulus, dynamin I is rephosphorylated. Rephosphorylation is essential for the next round of SVE (S2) to proceed (Fig. 4a). Roscovitine inhibited the rephosphorylation of dynamin I during repolarization (Fig. 4b, c), suggesting that Cdk5 is the endogenous dynamin I kinase in nerve terminals. These experiments also indicate that dynamin phosphorylation does not alter its interaction with the $\mathrm{SH} 3$ domain of its major partner, amphiphysin. Immunoblotting of nerve terminal lysates with phospho-specific antibodies confirmed that the endogenous phosphorylation sites on dynamin I were the same as those phosphorylated in vitro by Cdk5 (Ser 774 and Ser 778; Fig. 4d). The dephosphorylation at S1 detected by both antibodies was abolished by blocking calcineurin activity with cyclosporin A (data not shown). Cyclosporin A also inhibits SVE ${ }^{2,3,5}$, confirming that Ser 774 and Ser 778 are the physiologically relevant phosphorylation sites on dynamin I.

Phosphorylation of endogenous dynamin I on Ser 795 (the site for in vitro PKC phosphorylation) was not detectable with phospho-specific antibodies (Fig. 4d). This was not the result of an artefact in the immunoblotting protocol, as the same antibodies strongly detected dynamin I phosphorylated by PKC- $\alpha$ in vitro on the same blot (data not shown). Dynamin I is also an in vitro substrate for mitogen-activated protein kinase (MAPK), which targets [Ser/Thr]-Pro motifs such as Ser 774 and Ser 778 (ref. 14). However pre-incubation of nerve terminals with the MEK inhibitor PD98059 $(100 \mu \mathrm{M})$ had no effect on dynamin I rephosphorylation (see Supplementary Information, Fig. S3a). This strongly argues that $\mathrm{Cdk} 5$ is the endogenous kinase for dynamin I.

Next, we used a dominant-negative Cdk5 mutant to confirm the results with roscovitine. Green fluorescent protein (GFP)-tagged Cdk5 $5^{\mathrm{WT}}$ or Cdk5 $5^{\mathrm{DN}}$ was transfected into B104 neuroblastoma cells that had been allowed to differentiate for 10 days in the presence of cAMP. This allowed the cells to extend long processes containing multiple puncta that contact other neurites or cell bodies and which stain brightly for synapsin I or dynamin I (ref. 25). In cells transfected with GFP-Cdk5 5 WT, immunostaining for phospho-dynamin Ser 774 was unaltered (Fig. 5a-c) or increased (data not shown). However, in cells transfected with GFP-Cdk5 $5^{\mathrm{DN}}$, phospho-dynamin staining was greatly reduced in puncta (Fig. 5d-f) and in neurite processes (Fig. 5g-i), but not in untransfected cells in the same dishes or in the cell body (data not shown). In cells where recombinant GFP-Cdk $5^{\mathrm{DN}}$ had not yet migrated all the way into the distal tips of the neurites, phosphorylation staining was reduced in the proximal neurite, but not the distal portion of the same neurite (data not shown). The same results were obtained after co-transfection of primary cultures of cerebellar granule neurons (CGNs) with GFP and Cdk5 ${ }^{\mathrm{WT}}$ or GFP and $\mathrm{Cdk}^{\mathrm{DN}}$ (data not shown). This supports the pharmacological evidence that $\mathrm{Cdk} 5$ is the major dynamin kinase in neurons.

There are at least two dephosphin kinases, with the protein kinase for dynamin and synaptojanin being distinct from that for amphiphysin or AP180 (ref. 3). Amphiphysin is known to be an in vitro substrate for Cdk5 (refs 20,26), although the endogenous kinase in nerve terminals is unknown. We explored the apparent discrepancy between these observations. Using a double pull-down approach to simultaneously isolate phospho-AP180, synaptojanin, amphiphysin I and dynamin I from labelled synaptosomes, we found that roscovitine blocked synaptojanin rephosphorylation, but did not block rephosphorylation of amphiphysin or AP180 in the same synaptosomes (Fig. 4e, lanes 13 and 14). Therefore, amphiphysin and AP180 are not in vivo substrates for Cdk5, despite the in vitro phosphorylation of amphiphysin. Phosphorylation of synapsin I was relatively unaffected by roscovitine treatment (see Supplementary Information, Fig. S4). This confirms that there is at least one other dephosphin kinase apart from Cdk5 that phosphorylates AP180 and amphiphysin, and that Cdk5 is relatively specific for dynamin and synaptojanin. 
a

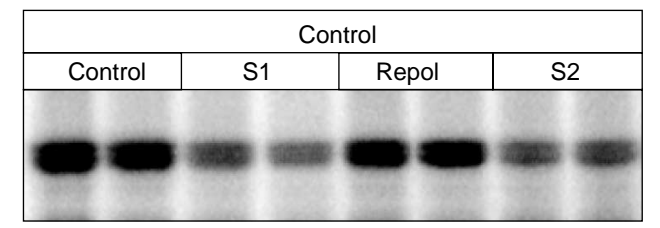

b
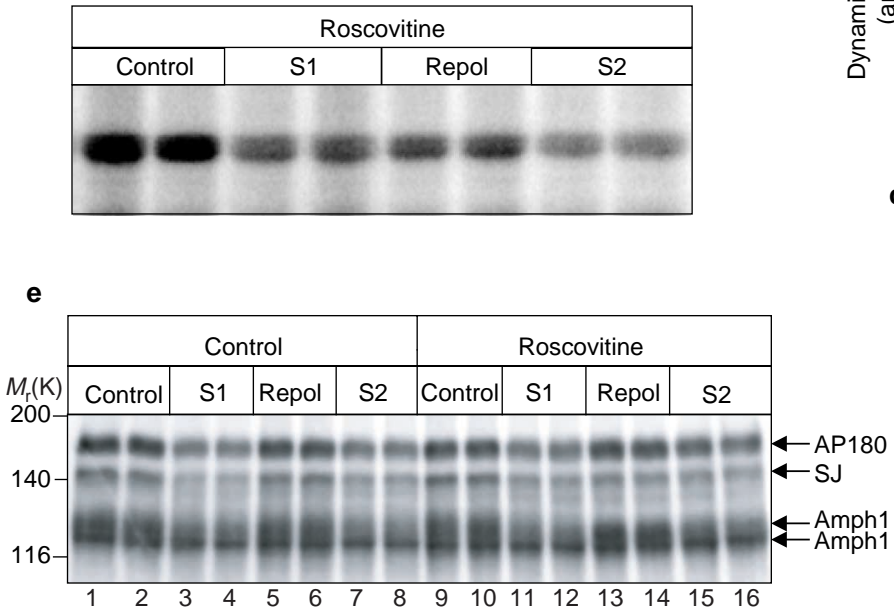

c

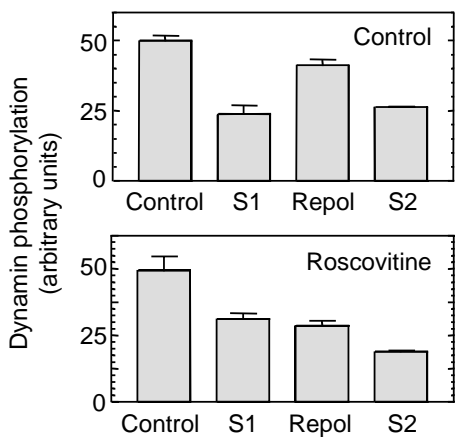

d

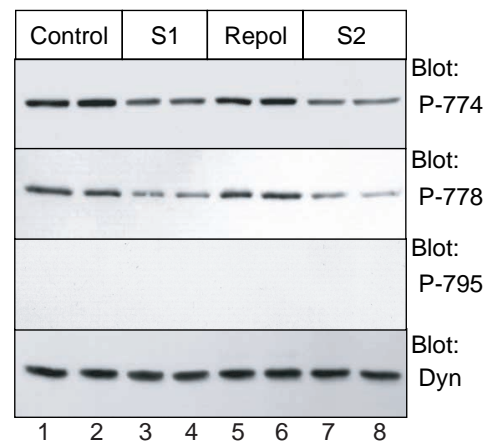

Figure 4 Roscovitine inhibits rephosphorylation of dynamin I in intact synaptosomes. (a) Dynamin I isolated from ${ }^{32} \mathrm{P}$-labelled intact synaptosomes is constitutively phosphorylated in resting nerve terminals (control), dephosphorylated after stimulation with $41 \mathrm{mM}$ potassium chloride (S1), rephosphorylated after potassium chloride removal (Repol) and is dephosphorylated a second time after stimulation (S2). Phospho-dynamin was isolated from the labelled synaptosomes by pull-downs with GST-amphiphysin-SH3 attached to GSH-sepharose. (b) Dynamin rephosphorylation during repolarization is inhibited by roscovitine ( $100 \mu \mathrm{M}$; Repol). (c) Quantification of data from $\mathbf{a}$ and $\mathbf{b}$ presented as storm phosphorimager units. Results are the mean \pm sem, $n=4$. (d) Synaptosomes were stimulated as above and proteins in the whole synaptosomal lysate were separated by SDS-PAGE and probed with antibodies to phospho-Ser 774, phospho-Ser 778, phospho-Ser 795 or to native dynamin I. Immunoblots are displayed. (e) Roscovitine blocks phosphorylation of synaptojanin, but not phosphorylation of amphiphysin or AP180. This experiment was identical to that in $\mathbf{a}$ and $\mathbf{b}$, except that four dephosphins (AP180, synaptojanin (SJ), amphiphysin 1 (a doublet) and dynamin 1 (data not shown)) and synapsin 1 (see Supplementary Information, Fig. S4) were extracted at the same time by pull-downs with a mix of GST- $\alpha$-adaptin appendage domain and GST-endophilin 1-SH3 domains attached to GSH-sepharose $(n=5$; each experiment performed in duplicate).

\section{Cdk5 activity is essential for SVE}

Calcineurin-mediated dephosphorylation of the dephosphins activates SVE in nerve terminals, and their subsequent rephosphorylation is required for maintaining repetitive rounds of the process ${ }^{3}$. To determine whether Cdk5 functions in SVE, we monitored exocytosis and endocytosis in nerve terminals incubated with roscovitine. Roscovitine had little effect on either the first (S1) or second (S2) round of exocytosis (calcium-dependent glutamate release) stimulated with $30 \mathrm{mM}$ potassium chloride (Fig. 6a, b). Roscovitine also had no significant effect on the initial round of SVE measured using a FM2-10 uptake assay $^{27}$ (Fig. 6d). This event represents S1 SVE, as uptake of the fluorescent dye FM2-10 occurs during S1 and accumulated dye is unloaded at S2 (Fig. 6c). The role of Cdk5 rephosphorylation in the second round of SVE was then examined. Roscovitine significantly inhibited S2 endocytosis (Fig. 6e), indicating that Cdk5 activity was required for the maintenance of SVE. 'Retrieval efficiency' is a quantitative measure of SVE that normalizes the amount of endocytosis for a given stimulus ${ }^{27}$. Retrieval efficiency is significantly decreased in roscovitine-treated nerve terminals at S2 $(34.2 \pm 0.1 \%$ inhibition $(n=7, P<0.001)$, but not at $\mathrm{S} 1$ (Fig. 6f). This effect is most probably the result of inactivation of Cdk5 by roscovitine, as the antagonist did not block dynamin I GTPase activity in vitro (data not shown). Inhibition of the MAPK pathway with $100 \mu \mathrm{M}$ PD98059 had no effect on SVE at either S1 or S2,

essentially ruling out a role for this pathway (see Supplementary Information, Fig. S3b).

To confirm a specific block of SVE by roscovitine, we used morphological analysis of nerve terminals, as previously performed for dynamin I mutations in Drosophila ${ }^{28}$. Subjecting untreated synaptosomes to two cycles of stimulus and recovery revealed no morphological changes (see Supplementary Information, Fig. S5 and Fig. 7a). Roscovitine treatment did not change the morphology of resting synaptosomes after one cycle of stimulus (S1; Fig. 7b) or recovery (data not shown). However, it had a marked effect on nerve terminal morphology at S2 (Fig. 7c). At S2, drug-treated synaptosomes were greatly depleted of synaptic vesicles, indicative of exocytosis in the absence of $\mathrm{SVE}^{28}$. Most terminals were devoid of synaptic vesicles and many exhibited significant plasma membrane ruffling and invaginations (Fig. 7c, arrows; also see Supplementary Information, Fig. S6), consistent with a block in SVE. These morphological observations, in conjunction with the functional assays of synaptic vesicle recycling, directly demonstrate that Cdk5 activity is necessary for SVE.

Finally, we explored the effect of overexpressing Cdk5 $5^{\mathrm{DN}}$ on SVE in nerve terminals of CGNs using the red-shifted styryl dye FM4-64. CGN nerve terminals co-transfected with GFP and Cdk5 5 WT demonstrated a stimulated loading and unloading of FM4-64 comparable with untransfected neurons (Fig. 8a-f). However neurons co-transfected with GFP 


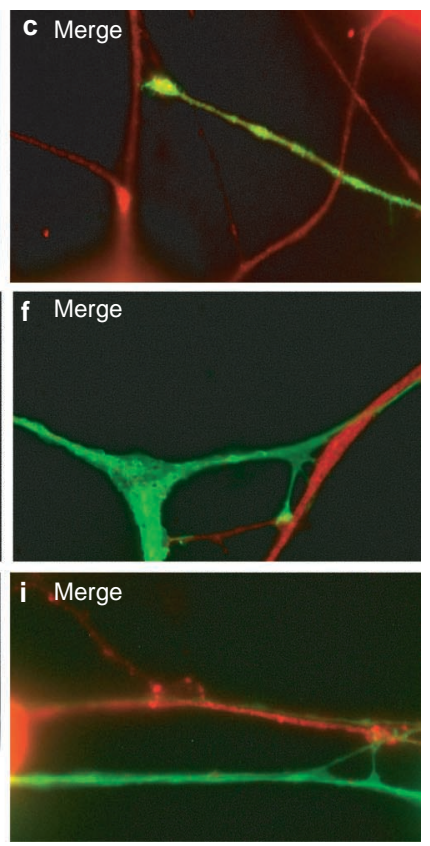

Figure 5 Dominant-negative Cdk5 inhibits dynamin phosphorylation in neurites. B104 rat brain neuroblastoma cells were differentiated for 10 days with CAMP and transfected with GFP-Cdk5WT (a-c) or GFP-Cdk5DN $(\mathbf{d}-\mathbf{i})$ for $5 \mathrm{~h}$. After $24 \mathrm{~h}$, the cells were fixed and co-stained with antibodies to dynamin phospho-Ser 774 ( $R$, red). (a-c) The neurite transfected with GFP-Cdk5 WT shows enrichment of Cdk5 in puncta and its presence throughout the body of the neurite (G, green). Phospho-dynamin staining in the transfected cell was equal in intensity to that of surrounding untransfected cells. (d-f) The transfected cell neurites show tips and termini with enriched GFP-Cdk5 ${ }^{\mathrm{DN}}$, which make contacts with the cell body and neurites of an underlying non-transfected cell. The transfected cell neurite and puncta show almost no staining for phospho-dynamin. (g-i) Phosphodynamin staining is punctate and present in neurites of non-transfected cells, but greatly diminished in the neurites of cells transfected with GFP-Cdk5DN. Scale: panel widths are $109 \mu \mathrm{m}$ in a-c, $105 \mu \mathrm{m}$ in $\mathbf{d}-\mathbf{f}$ and $67 \mu \mathrm{m}$ in $\mathbf{g}-\mathbf{i}$. and $\mathrm{Cdk} 5^{\mathrm{DN}}$ (Fig. $8 \mathrm{~g}-1$ ) demonstrated a greatly reduced loading of FM4-64 when compared with neighbouring untransfected neurons (Fig. 8i). Quantitative analysis of the stimulated loading and unloading of FM4-64 showed that the total synaptic vesicle recycling pool was reduced by approximately $50 \%$ in CGNs transfected with Cdk $5^{\text {DN }}$ (Fig. $8 \mathrm{~m}, \mathrm{n} ; 48.8 \pm 4.5 \%$ of control). To establish whether the reduction in the total synaptic vesicle recycling pool was the result of a decrease in the number of synaptic vesicles undergoing endocytosis or exocytosis, we examined the kinetics of FM4-64 unloading after stimulation with potassium chloride. The kinetics of FM4-64 unloading was not significantly different between untransfected CGNs and those transfected with $\mathrm{Cdk} 5^{\mathrm{DN}}$ (data not shown). Thus, the reduction in the total synaptic vesicle recycling pool was mediated by an inhibition of SVE and not exocytosis, confirming that Cdk5 is required for SVE in primary neurons, in addition to synaptosomes.

\section{DISCUSSION}

In this study, we demonstrated two new interrelated functions for Cdk5 in neuronal function. First, $\mathrm{Cdk} 5$ is identified as the protein kinase that rephosphorylates dynamin I after termination of SVE in nerve terminals. This is the first demonstration of an in vivo dephosphin kinase. Second, we have shown that Cdk5-dependent rephosphorylation of dynamin I (and possibly synaptojanin) is essential for SVE. Phosphorylation of AP180 and amphiphysin was shown not to be mediated by Cdk5 in vivo, revealing the existence of at least one additional dephosphin kinase. Thus, the protein kinase activity previously demonstrated to be essential for multiple rounds of $\mathrm{SVE}^{3}$ has now been identified as Cdk5.
The demonstration that $\mathrm{Cdk} 5$ is the endogenous dynamin I kinase resolves many of the apparent inconsistencies surrounding PKC as the candidate dynamin I kinase. Dynamin I is phosphorylated by calciumdependent PKCs (cPKCs) in vitro with rapid kinetics ${ }^{7,29}$ and relatively selective PKC inhibitors block its rephosphorylation in nerve terminals ${ }^{3,30}$. However other known PKC substrates are phosphorylated during nerve terminal stimulation, which is the reverse of the situation with dynamin I. Also, rephosphorylation of dynamin I occurs after, not during, increases in nerve terminal intracellular calcium levels, suggesting that cPKCs are not involved ${ }^{31}$. Finally, the subcellular location of cPKCs during SVE is the opposite of that expected if it were the dynamin I kinase $\mathrm{e}^{5,7,29}$. In this study, we effectively rule out $\mathrm{PKC}$, as its in vitro phosphorylation site is not phosphorylated in vivo. In contrast, Cdk5 fulfils all of the criteria expected for a dynamin I kinase. First, it is a tonically active protein kinase enriched in nerve terminals and its active form is localized to membrane fractions ${ }^{17}$. Second, it phosphorylates dynamin I in vitro on sites identical to the endogenous sites on dynamin I. Finally, selective antagonists of Cdk5 inhibit dynamin I rephosphorylation in nerve terminals, whereas a dominant-negative Cdk5 mutant blocks phosphorylation of dynamin in nerve terminals.

The role of Cdk5 and its activator p35 is well established in neuronal development, with defined roles in neuronal migration, differentiation and synaptogenesis ${ }^{32}$. Cdk5 knockout mice exhibit perinatal lethality and lack both cortical laminar structure and cerebellar foliation $^{33}$. P35 knockout mice survive past birth, but also show abnormal layering of the cortex and are prone to seizures and early mortality ${ }^{34}$. However, our results reveal that Cdk5 is a significant factor in SVE in the mature synapse. Other evidence supports a role for Cdk5 at the 
a

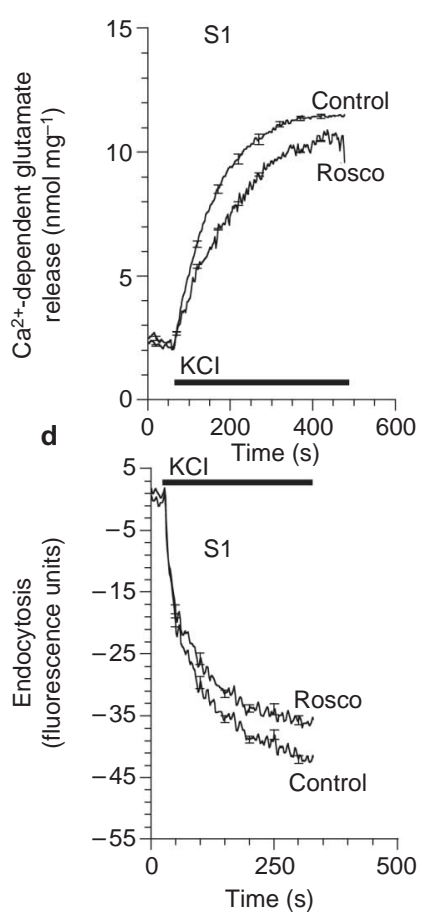

b
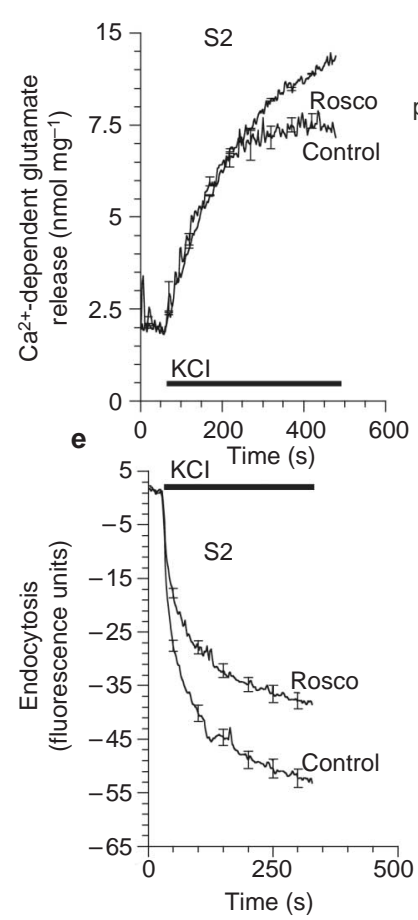

c

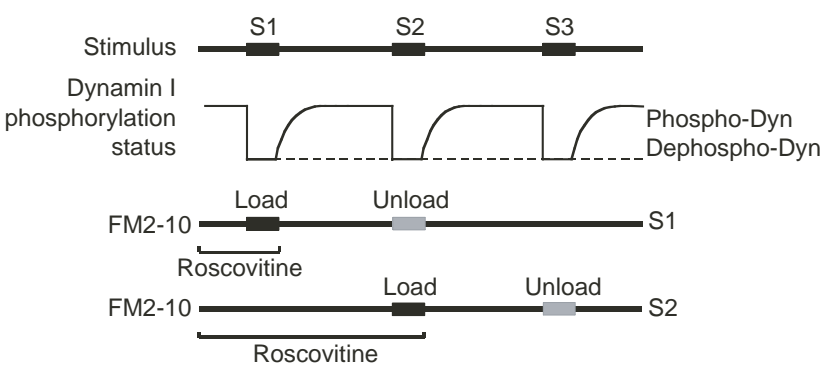

$\mathbf{f}$

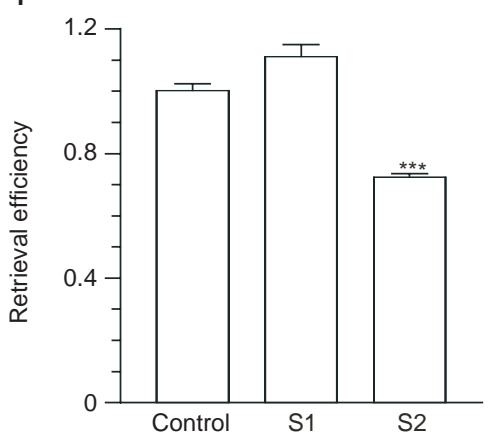

Figure 6 Cdk5 activity is required for SVE in nerve terminals. (a, b) Synaptosomes were incubated with or without roscovitine $(100 \mu \mathrm{M})$ for 15 min and subjected to two cycles of stimulus and repolarization. Exocytosis (calcium-dependent glutamate release) was stimulated by $30 \mathrm{mM}$ potassium chloride at $\mathrm{S} 1$, as shown in $\mathbf{a}$, and $\mathrm{S} 2$, as shown in $\mathbf{b}$, in the presence (Rosco) or absence (control) of roscovitine. (c) Protocol for the SVE assay. (d, e) Endocytosis (calcium-dependent uptake and release of FM2 10) at S1, as shown in $\mathbf{d}$, and S2, as shown in e, in the presence or absence of roscovitine. The traces are averages of at least three experiments \pm sem. (f) Retrieval efficiency (endocytosis divided by exocytosis at $120 \mathrm{~s}$ ) at S1 was unaffected by roscovitine treatment, whereas at S2 it was inhibited by $34.2 \%(n=7) ;{ }^{* * *} P<0.001$. mature pre-synaptic terminal. Cdk5 is expressed predominantly in post-mitotic neurons, but not in glial cells or mitotically active cells ${ }^{35}$. It is also enriched in mature nerve terminals ${ }^{17}$. Cdk5 also phosphorylates nerve terminal proteins in vitro that are implicated in synaptic vesicle recycling, such as synapsin I (ref. 18), amphiphysin I (ref. 20), munc-18 (ref. 19) and DARPP-32 (ref. 36), suggesting possible multiple roles for the protein kinase in synaptic vesicle recycling. Inhibition of Cdk5 has produced conflicting results when examining transmitter release from secretory cells and neurones ${ }^{17,37,38}$. Roscovitine also has Cdk5-independent effects on voltage-dependent P/Q-type calcium channels ${ }^{39}$. By slowing calcium channel inactivation kinetics, roscovitine increased calcium influx (and thus neurotransmitter release) from both synaptosomes and primary neuronal cultures ${ }^{17,39}$. We did not observe this facilitation of glutamate release, possibly because our method of stimulation relies preferentially on non-inactivating calcium channels ${ }^{40}$. Thus, using our stimulation protocol we can examine the effect of roscovitine on SVE without the complication of additional effects on synaptic vesicle exocytosis. Using Cdk5 $5^{\mathrm{DN}}$ as an alternative approach to roscovitine treatment, we also found that SVE was blocked in CGNs. Thus, our results rule out an artefact in the use of roscovitine to study SVE.

We observed a selective inhibition of the second round of SVE in synaptosomes by roscovitine treatment, indicating that Cdk5 is controlling endocytosis by rephosphorylation of a specific subset of dephosphins after nerve terminal stimulation. The first round of SVE removes endogenous phosphate from dynamin, before rephosphorylation. The situation differs in cultured neurons transfected with
Cdk5 $5^{\mathrm{DN}}$, where the background phosphorylation of dynamin is reduced before examination of SVE. Protein phosphorylation is currently thought to control SVE through two major mechanisms ${ }^{5}$ : dephosphorylation-dependent association of dephosphins with membrane lipids ${ }^{7}$ and/or themselves ${ }^{4-43}$. Our results support the former mechanism, as phosphorylation by Cdk5 prevented association of dynamin with lipids in vitro, correlating with a cytosolic location for phospho-dynamin in synaptosomes. The regulation of dynamin I phosphorylation by amphiphysin I is of particular interest. One of the endocytic protein complexes that might assemble on the nerve terminal membrane after dephosphorylation includes dynamin I, amphiphysin I/II and endophilin ${ }^{43}$. This complex might also include Cdk5, through an interaction of amphiphysin I with p35 (ref. 20). Our results suggest an interesting mechanism whereby Cdk5 indirectly controls dynamin I phosphorylation during SVE. In this model, the endocytic machinery recruits Cdk5 through its interaction with amphiphysin I while limiting its available substrate, as the dynamin I-amphiphysin I interaction prevents dynamin I from being phosphorylated. After SVE is complete and the complex dissociates, dynamin I can be rephosphorylated by Cdk5 for the next round of SVE. Moreover, the phosphorylation status of amphiphysin I, but not dynamin I, controls the interaction between these two proteins in vitro, as amphiphysin I that is phosphorylated by an unidentified brain protein kinase cannot bind dynamin I (ref. 43), but Cdk5 phosphorylation of dynamin I has no effect ${ }^{2,43}$. Amphiphysin I is an in vitro substrate for Cdk5, but we show it is not an endogenous substrate in nerve terminals. It is possible that phosphorylation by a second dephosphin kinase 


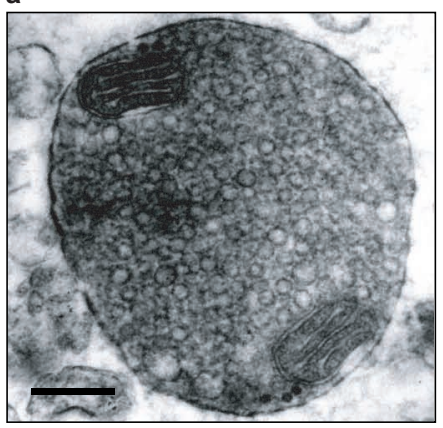

S2 Control

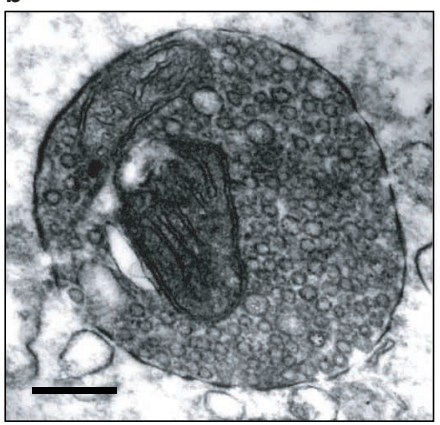

S1 Rosco

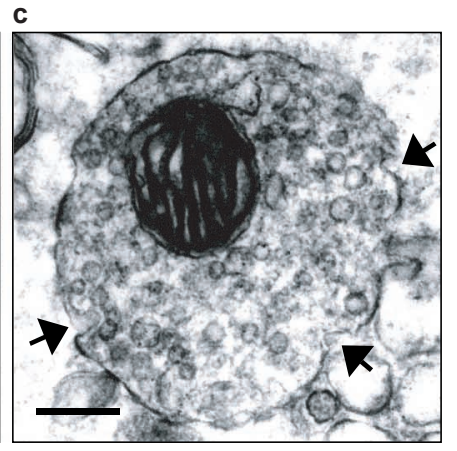

S2 Rosco
Figure 7 Inhibition of Cdk5 produces a morphologically distinct block in SVE. Electron micrographs of synaptosomes incubated with or without 100 $\mu \mathrm{M}$ roscovitine. (a) Typical morphology found in control untreated synaptosomes at rest, S1, repolarization or S2. The terminals are filled with synaptic vesicles and contain a mitochondrial profile and a smooth, sealed plasma membrane. (b) Even after depolarization in the presence of roscovitine (S1), the morphology is essentially unaltered. (c) At S2 with roscovitine treatment, the synaptosome has a greatly depleted vesicle population and numerous plasma membrane invaginations (arrows) suggestive of an endocytic block. Scale bar represents $0.2 \mu \mathrm{m}$. keeps it separated from dynamin I until SVE is initiated.

In conclusion, we propose that Cdk5 is essential for SVE in the mature neuron. Our results establish $\mathrm{Cdk} 5$ as the in vivo protein kinase for dynamin I and synaptojanin in nerve terminals, representing the first demonstration of an endogenous dephosphin kinase. In addition, we establish that there is at least one other endogenous dephosphin kinase that targets amphiphysin and/or AP180. We propose that the rephosphorylation of dynamin I by Cdk 5 is essential for repeated cycles of SVE and that this designates a new biological role for Cdk5 in the regulation of synaptic transmission.

\section{METHODS}

Chemicals, constructs and antibodies. Plasmids: GST-dynamin I-PRD, amphiphysin I- and II-SH3 were from P. De Camilli (Yale, New Haven, CT), GST-Cdk5 was from J. Wang (Hong Kong University, Hong Kong), GST-p25 was from L.-H. Tsai (Harvard Medical School, Boston, MA), the SH3 domains of Grb2 and p85 were as previously described ${ }^{44}$, GST- $\alpha$-adaptin appendage domain was from R. Anderson (Dallas, TX), GST-endophilin 1-SH3 was from P. McPherson (McGill, Canada) and transfection plasmids for GFP-Cdk5 $\left(\mathrm{Cdk} 5^{\mathrm{WT}}\right.$ or $\left.\mathrm{Cdk} 5^{\mathrm{DN}}\left(\mathrm{Cdk} 5^{\mathrm{K} 33 \mathrm{~T}}\right)\right)$ or untagged Cdk5 (WT or DN) were from P. Zelenka (NIH, Bethesda, MD) ${ }^{45}$ or S. van den Heuvel (Harvard Medical School ${ }^{46}$. Affinity purified rat His-tagged PKC- $\alpha$ was expressed in baculovirusinfected Sf9 cells (recombinant virus provided by T. Biden, Garvan Institute, Australia). Antibodies to phospho-Ser 795 were from Santa Cruz Biotechnology (Santa Cruz, CA). Phospho-specific antibodies to phospho-Ser 774 and phospho-Ser 778 were raised in sheep against synthetic phosphopeptides PAGRRSPTSC and CTSSPTPQR, respectively (where the underlined Ser represents phospho-Ser). The peptides were conjugated to diphtheria toxoid by the cysteine residue that was added during synthesis. The antibodies were specific for phospho-Ser residues on dynamin I as neither detected other Cdk5 substrates, such as synapsin I (ref. 18), amphiphysin I (ref. 20), histone or proteins in cytosolic brain extracts (data not shown).

Protein purification. Dynamin I was purified from sheep brain ${ }^{7,47}$ for in vitro studies and from rat brain synaptosomes for in vivo studies. Bacterial plasmid constructs were expressed in Escherichia coli according to the manufacturers' instructions. GST-Cdk5 and GST-p25 expressed in bacteria and cell pellets from the two constructs were mixed, co-lysed and co-affinity purified using GSH-Sepharose. Next, GSH-Sepharose with bound GST-Cdk5 and GST-p25 was incubated with rat brain cytosol for $2 \mathrm{~h}$ at $4{ }^{\circ} \mathrm{C}$ before extensive washing, elution with $10 \mathrm{mM}$ reduced GSH in $50 \mathrm{mM}$ Tris- $\mathrm{HCl}$ at $\mathrm{pH} 8.0$, dialysis to remove $\mathrm{GSH}$ and storage at $-20^{\circ} \mathrm{C}$.

In vitro phosphorylation. Dynamin I was phosphorylated with recombinant
PKC- $\alpha$ or Cdk 5 for $10 \mathrm{~min}^{7}$. Samples were analysed by SDS polyacrylamide gel electrophoresis (SDS-PAGE) and autoradiography. Phospho-dynamin I was analysed by peptide mapping using V8 protease and phospho-amino acid analysis by thin layer electrophoresis ${ }^{12}$. PKC assays ${ }^{7}$ were performed in the absence or presence of roscovitine. Dynamin I was phosphorylated stoichiometrically with PKC- $\alpha$ or Cdk5 (ref. 7) for 60 min before MALDI-TOF MS analysis.

${ }^{32} \mathrm{P}$ labelling of synaptosomes and pull-down experiments. P2 synaptosomes were prepared from rat brain, labelled with ${ }^{32} \mathrm{P}$ (refs 3, 12), pre-incubated for $15 \mathrm{~min}$ at $37^{\circ} \mathrm{C}$ in the absence or presence of $100 \mu \mathrm{M}$ roscovitine and depolarized with $41 \mathrm{mM}$ potassium chloride (S1). Synaptosomes were repolarized in the presence or absence of roscovitine for $5 \mathrm{~min}$ before a second stimulation with potassium chloride (S2). Synaptosomes at each point (control, S1, repolarization and S2) were lysed in $25 \mathrm{mM}$ Tris- $\mathrm{HCl}$ at $\mathrm{pH} 7.4$ containing $1 \%$ Triton X-100, $150 \mathrm{mM}$ sodium chloride, $1 \mathrm{mM}$ EGTA, $2 \mathrm{mM}$ EDTA, $50 \mathrm{mM}$ sodium fluoride, $20 \mu \mathrm{g} \mathrm{ml}^{-1}$ leupeptin, $1 \mathrm{mM}$ phenyl methylsulphonyl fluoride (PMSF) and EDTA-free protease inhibitor cocktail (Calbiochem-Novabiochem, Kilsyth, Victoria, Australia) and centrifuged at $20,442 \mathrm{~g}$ for $15 \mathrm{~min}$ at $4{ }^{\circ} \mathrm{C}$. Dynamin I was affinity purified from the supernatant using the GST-amphiphysin I-SH3 domain (Fig. 3 or Fig. $4 \mathrm{a}-\mathrm{c}$ ) bound to $\mathrm{GSH}-$ sepharose for $1 \mathrm{~h}$ at $4{ }^{\circ} \mathrm{C}^{3}$. The washed beads were heated in sample buffer and released proteins were separated by SDS-PAGE. In a double pull-down experiment, four dephosphins and synapsin I were simultaneously affinity purified in the same fashion using a mixture of two GST fusion proteins. Recombinant GST- $\alpha$-adaptin appendage domain ${ }^{48}$ and GST-endophilin 1-SH3 domain ${ }^{49}$ were coupled to GSH-Sepharose and beads containing equal amounts of recombinant protein were mixed. The mixture used to simultaneously pull-down AP180, synaptojanin, amphiphysin I, dynamin I and synapsin I from the ${ }^{32} \mathrm{P}$-labelled synaptosomes (Fig. $4 \mathrm{e}$ ). The identity of each protein was confirmed both by MALDI-TOF mass spectrometry and by immunoblotting.

Phosphopeptide analysis by MALDI-TOF MS. Phospho-dynamin I from ${ }^{32} \mathrm{P}$ labelled synaptosomes and dynamin I phosphorylated by Cdk5 and PKC in vitro were excised from gels and submitted to in-gel tryptic digestion. The peptide mixtures were purified using $\mathrm{Fe}^{3+}$-IMAC agarose columns and the affinitybound phosphopeptides were not eluted from the agarose media before MS analysis ${ }^{50}$. Bound peptides were analysed by MALDI-TOF MS before and after alkaline phosphatase treatment ${ }^{7}$.

Western blotting. P2 synaptosomes prepared from rat brain ${ }^{12}$ were lysed in SDS sample buffer, heated to $100^{\circ} \mathrm{C}$ for $2 \mathrm{~min}$, centrifuged at $20,442 \mathrm{~g}$ for $5 \mathrm{~min}$ at $4^{\circ} \mathrm{C}$ and the resulting supernatant separated by SDS-PAGE on $12 \%$ acrylamide gels. Western blots were analysed by ECL using the Pierce super-signal kit.

Endocytosis and exocytosis assays. The exocytosis (glutamate release) and endocytosis assays were performed using Percoll-purified synaptosomes, as 

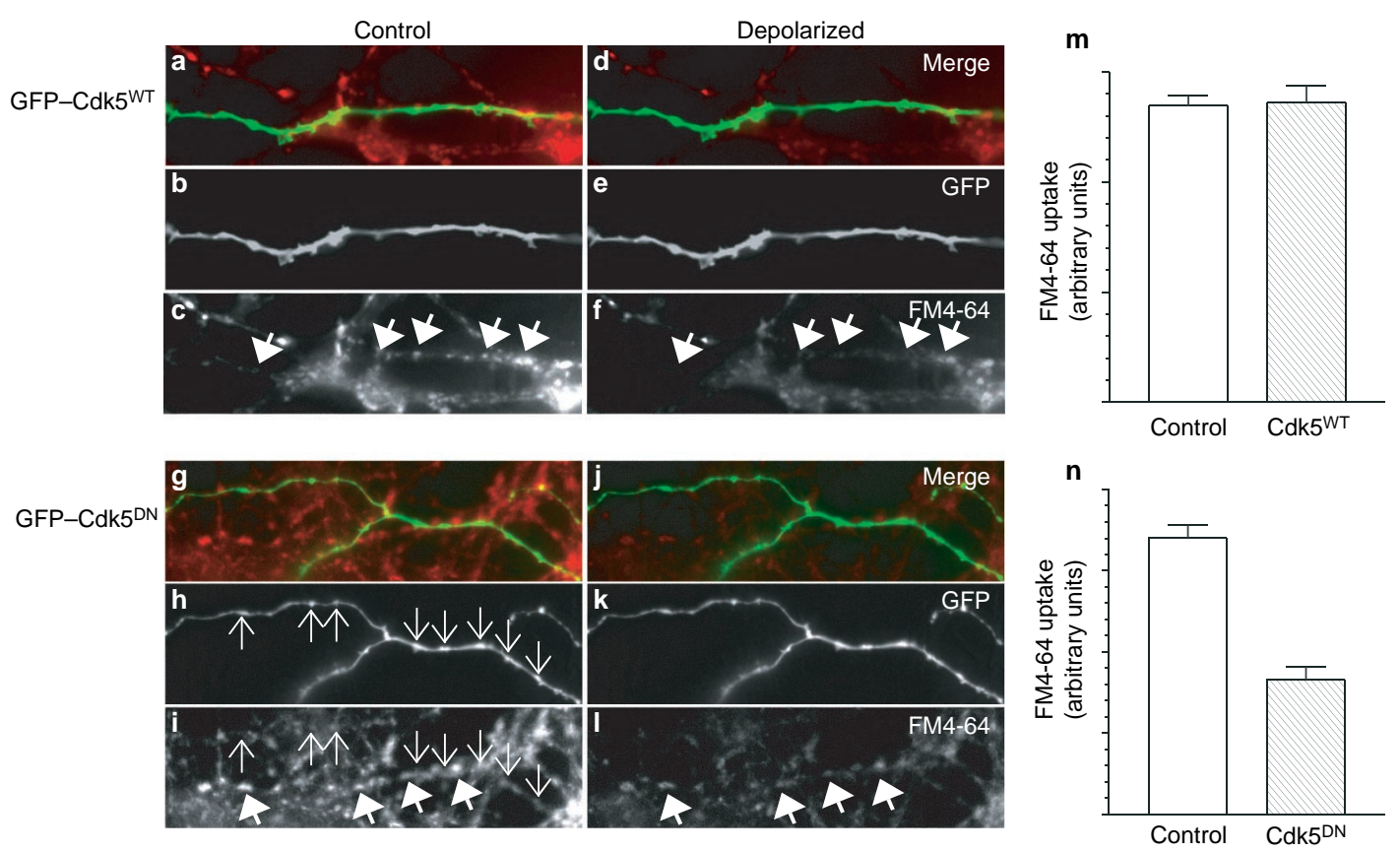

Figure $8 \mathrm{Cdk5} 5^{\mathrm{DN}}$ blocks SVE in cerebellar granule neurons. (a-f) Primary cultures of CGNs were co-transfected with GFP and Cdk5 $5^{\mathrm{WT}}$ (green) and stimulated uptake of FM4-64 (red) was visualized by fluorescence microscopy. To confirm that the red puncta (thick arrows) represent sites of SVE (c), CGNs were depolarized to unload the accumulated FM4-64. The fluorescence of all puncta was reduced (f). (g-I) CGNs co-transfected with GFP and Cdk5 ${ }^{\mathrm{DN}}$ accumulated considerably less FM4-64, indicating a block in SVE (thin arrows in $\mathbf{g - i}$ ). The reduction in FM4-64 loading was not caused by effects on exocytosis, as the limited amount of accumulated dye could be released during a second stimulation (I). Untransfected neurons

described previously ${ }^{3}$. The protocol for the SVE assay is shown in Fig. 6c. Briefly, FM2-10 was accumulated into synaptic vesicles during SVE by stimulation with $30 \mathrm{mM}$ potassium chloride in the presence of roscovitine (S1) or were stimulated, repolarized and stimulated again in the presence of roscovitine (S2). The amount of FM2-10 accumulated by SVE was estimated by unloading the dye with a subsequent potassium chloride stimulus (S2 or S3), Roscovitine did not affect the ability of this standard pulse of potassium chloride to evoke release of FM2-10-labelled vesicles, as calcium-dependent glutamate release was unaffected for S1, S2 and S3 (data not shown). Retrieval efficiency ${ }^{27}$ was calculated as endocytosis divided by exocytosis, where endocytosis is total accumulated FM2-10 and exocytosis is calcium-dependent glutamate release after 2 min of stimulation.

Electron microscopy. Percoll-purified synaptosomes were prepared as previously described ${ }^{3}$. Synaptosomes at four different states (control, S1, repolarized and S2), with and without roscovitine treatment, were fixed in 5\% glutaraldehyde in phosphate-buffered saline and processed for electron microscopy by conventional methods.

Note: Supplementary Information is available on the Nature Cell Biology website.

\section{ACKNOWLEDGEMENTS}

This work was supported by grants from the Australian National Health and Medical Research Council (NHMRC to P.J.R.), the Cunningham Trust and Wellcome Trust (M.A.C.) and the Danish Natural Science Research Council (M.R.L.). We thank P. Rowe for critical reading of the manuscript. We also thank A. Quan (Children's Medical Research Institute), B. Hanna (Children's Medical Research Institute) and L. Deer (Institute of Clinical Pathology and Medical Research, Westmead Hospital) for valuable technical assistance. In addition, we also thank our colleagues listed in the constructs section for the plasmids required for this project. loaded and unloaded FM4-64 normally (thick arrows $\mathbf{i}-\mathbf{I})$. ( $\mathbf{m}, \mathbf{n}$ ) The total amount of activity-dependent FM4-64 accumulation is displayed in CGNs co-transfected with GFP and Cdk5WT (m) or GFP and Cdk5DN (n). The bar graph represents the maximal amount of FM4-64 accumulated and then unloaded during stimulation with $50 \mathrm{mM}$ potassium chloride. Control indicates FM4-64 loading in neighbouring CGN nerve terminals within the same field of view. Data are taken from 3-5 experiments $(n=28$ nerve terminals for $\mathrm{Cdk} 5{ }^{\mathrm{WT}} ; n=83$ nerve terminals for $\mathrm{Cdk} 5^{\mathrm{WT}}$ controls; $n=55$ nerve terminals forCdk $5^{\mathrm{DN}} ; n=166$ nerve terminals for $\mathrm{Cdk} 5^{\mathrm{DN}}$ controls; all \pm sem).

\section{COMPETING FINANCIAL INTERESTS}

The authors declare that they have no competing financial interests.

Received 18 September 2002 ; Accepted 20 June 2003; Published online: 13 July 2003; DOI: 10.1038/ncb1020.

1. Liu, J. P., Sim, A. T. R. \& Robinson, P. J. Calcineurin inhibition of dynamin I GTPase activity coupled to nerve terminal depolarization. Science 265, 970-973 (1994).

2. Marks, B. \& McMahon, H. T. Calcium triggers calcineurin-dependent synaptic vesicle recycling in mammalian nerve terminals. Curr.Biol. 8, 740-749 (1998).

3. Cousin, M. A., Tan, T. C. \& Robinson, P. J. Protein phosphorylation is required for endocytosis in nerve terminals. Potential role for the dephosphins dynamin I and synaptojanin, but not AP180 or amphiphysin. J. Neurochem. 76, 105-116 (2001).

4. Cousin, M. A. Synaptic vesicle endocytosis: calcium works overtime in the nerve terminal. Mol. Neurobiol. 22, 115-128 (2000).

5. Cousin, M. A. \& Robinson, P. J. The dephosphins: Dephosphorylation by calcineurin triggers synaptic vesicle endocytosis. Trends Neurosci. 24, 659-665 (2001).

6. Wenk, M. R. et al. PIP Kinase I $\gamma$ is the major $\mathrm{PI}(4,5) \mathrm{P}_{2}$ synthesizing enzyme at the synapse. Neuron 32, 79-88 (2001).

7. Powell, K. A. et al. Phosphorylation of dynamin I on Ser-795 by protein kinase C blocks its association with phospholipids. J. Biol. Chem. 275, 11610-11617 (2000).

8. Marks, B. et al. GTPase activity of dynamin and resulting conformation change are essential for endocytosis. Nature 410, 231-235 (2001).

9. Shupliakov, O. et al. Synaptic vesicle endocytosis impaired by disruption of dynaminSH3 domain interactions. Science 276, 259-263 (1997).

10. Gad, H. et al. Fission and uncoating of synaptic clathrin-coated vesicles are perturbed by disruption of interactions with the SH3 domain of endophilin. Neuron 27, 301-312 (2000).

11. Robinson, P. J. Dephosphin, a 96,000 dalton substrate of protein kinase $C$ in synaptosomal cytosol is phosphorylated in intact synaptosomes. FEBS Lett. 282, 388-392 (1991).

12. Robinson, P. J. et al. Dynamin GTPase regulated by protein kinase $\mathrm{C}$ phosphorylation in nerve terminals. Nature 365, 163-166 (1993).

13. Hosoya, H. et al. Phosphorylation of dynamin by cdc2 kinase. Biochem. Biophys. Res Commun. 202, 1127-1133 (1994).

14. Earnest, S., Khokhlatchev, A., Albanesi, J. P. \& Barylko, B. Phosphorylation of dynamin by ERK2 inhibits the dynamin-microtubule interaction. FEBS Lett. 396, 
62-66 (1996).

15. Chen-Hwang, M.-C., Chen, H.-R., Elzinga, M. \& Hwang, Y.-W. Dynamin is a minibrain kinase/Dyrk1A substrate. J. Biol. Chem. 277, 17597-17604 (2002).

16. Songyang, Z. et al. A structural basis for substrate specificities of protein Ser/Thr kinases: primary sequence preference of casein kinases I and II, NIMA, phosphorylase kinase, calmodulin-dependent kinase II, CDK5, and Erk1. Mol. Cell. Biol. 16, 6486-6493 (1996).

17. Tomizawa, K. et al. Cdk5/p35 regulates neurotransmitter release through phosphorylation and downregulation of $\mathrm{P} / \mathrm{Q}$-type voltage-dependent calcium channel activity. J. Neurosci. 22, 2590-2597 (2002)

18. Matsubara, M. et al. Site-specific phosphorylation of synapsin I by mitogen-activated protein kinase and Cdk5 and its effects on physiological functions. J. Biol. Chem. 271, 21108-21113 (1996).

19. Shuang, R. Q. et al. Regulation of Munc-18 syntaxin $1 \mathrm{~A}$ interaction by cyclin-dependent kinase 5 in nerve endings. J. Biol. Chem. 273, 4957-4966 (1998)

20. Floyd, S. R. et al. Amphiphysin binds the cdk5 regulatory subunit p35 and is phosphorylated by cdk5 and cdc2. J. Biol. Chem. 276, 8104-8110 (2001).

21. Hill, E., van der, K. J., Downes, C. P. \& Smythe, E. The role of dynamin and its binding partners in coated pit invagination and scission. J. Cell Biol. 152, 309-324 (2001).

22. Grabs, D. et al. The SH3 domain of amphiphysin binds the proline-rich domain of dynamin at a single site that defines a new SH3 binding consensus sequence. J. Biol. Chem. 272, 13419-13425 (1997).

23. Meijer, L. et al. Biochemical and cellular effects of roscovitine, a potent and selective inhibitor of the cyclin-dependent kinases cdc2, cdk2 and cdk5. Eur. J. Biochem. 243, 527-536 (1997).

24. Davies, S. P., Reddy, H., Caivano, M. \& Cohen, P. Specificity and mechanism of action of some commonly used protein kinase inhibitors. Biochem. J. 351, 95-105 (2000).

25. Cousin, M. A. et al. Synapsin I-associated phosphatidylinositol 3-kinase mediates synaptic vesicle delivery to the readily releasable pool. J. Biol. Chem. (cited 16 May 2003) DOI: 10.1074/jbc.M302386200 (2003).

26. Rosales, J. L., Nodwell, M. J., Johnston, R. N. \& Lee, K. Y. Cdk5/p25(nck5a) interaction with synaptic proteins in bovine brain. J. Cell. Biochem. 78, 151-159 (2000).

27. Cousin, M. A. \& Robinson, P. J. Ca ${ }^{2+}$ inhibition of dynamin arrests synaptic vesicle recycling at the active zone. J. Neurosci. 20, 949-957 (2000).

28. Koenig, J. H. \& Ikeda, K. Disappearance and reformation of synaptic vesicle membrane upon transmitter release observed under reversible blockage of membrane retrieval. J. Neurosci. 9, 3844-3860 (1989).

29. Liu, J. P., Powell, K. A., Südhof, T. C. \& Robinson, P. J. Dynamin I is a $\mathrm{Ca}^{2+}{ }_{- \text {sensitive }}$ phospholipid-binding protein with very high affinity for protein kinase C. J. Biol. Chem. 269, 21043-21050 (1994).

30. Robinson, P. J. Differential stimulation of protein kinase $C$ activity by phorbol ester or calcium/phosphatidylserine in vitro and in intact synaptosomes. J. Biol. Chem. 267 21637-21644 (1992)

31. Robinson, P. J., Liu, J. P., Powell, K. A., Fykse, E. M. \& Südhof, T. C. Phosphorylation of dynamin I and synaptic vesicle recycling. Trends Neurosci. 17, 348-353 (1994).
32. Dhavan, R., Tsai, L. H. \& Tsai, L. H. A decade of cdk5. Nature Rev. Mol. Cell Biol. 2, 749-759 (2001).

33. Ohshima, T. et al. Targeted disruption of the cyclin-dependent kinase 5 gene results in abnormal corticogenesis, neuronal pathology and perinatal death. Proc. Natl Acad. Sci. USA 93, 11173-11178 (1996).

34. Kwon, Y. T., Tsai, L. H. \& Crandall, J. E. Callosal axon guidance defects in $p 35(-/-)$ mice. J. Comp. Neurol. 415, 218-229 (1999).

35. Tsai, L. H., Takahashi, T., Caviness, V. S. J. \& Harlow, E. Activity and expression pattern of cyclin-dependent kinase 5 in the embryonic mouse nervous system. Development 119, 1029-1040 (1993).

36. Bibb, J. A. et al. Effects of chronic exposure to cocaine are regulated by the neuronal protein Cdk5. Nature 410, 376-380 (2001).

37. Fletcher, A. I. et al. Regulation of exocytosis by cyclin-dependent kinase 5 via phosphorylation of munc18. J. Biol. Chem. 274, 4027-4035 (1999).

38. Li, B. S. et al. Regulation of NMDA receptors by cyclin-dependent kinase-5. Proc. Nat Acad. Sci. USA 98, 12742-12747 (2001)

39. Yan, Z., Chi, P., Bibb, J. A., Ryan, T. A. \& Greengard, P. Roscovitine: a novel regulator of $P / Q$-type calcium channels and transmitter release in central neurons. J. Physiol. 540, 761-770 (2002).

40. Nicholls, D. G. The glutamatergic nerve terminal. Eur. J. Biochem. 212, 613-631 (1993).

41. Wilde, A. \& Brodsky, F. M. In vivo phosphorylation of adaptors regulates their interaction with clathrin. J. Cell Biol. 135, 635-645 (1996).

42. Kariya, K. et al. Regulation of complex formation of POB1/epsin/adaptor protein complex 2 by mitotic phosphorylation. J. Biol. Chem. 275, 18399-18406 (2000).

43. Slepnev, V. I., Ochoa, G. C., Butler, M. H., Grabs, D. \& DeCamilli, P. Role of phosphorylation in regulation of the assembly of endocytic coat complexes. Science $\mathbf{2 8 1}$, 821-824 (1998).

44. Rasmussen, R. K., Rusak, J., Price, G., Robinson, P. J. \& Dorow, D. S. Mixed lineage kinase 2-SH3 domain binds dynamin and greatly enhances GTPase activation by phospholipid. Biochem. J. 335, 119-124 (1998).

45. Negash, S., Wang, H. S., Gao, C., Ledee, D. \& Zelenka, P. Cdk5 regulates cell-matrix and cell-cell adhesion in lens epithelial cells. J. Cell Sci. 115, 2109-2117 (2002).

46. van den Heuvel, S. \& Harlow, E. Distinct roles for cyclin-dependent kinases in cell cycle control. Science 262, 2050-2054 (1993).

47. Stowell, M. H. Marks, B., Wigge, P. \& McMahon, H. T Nucleotide-dependent conformational changes in dynamin: evidence for a mechanochemical molecular spring. Nature Cell Biol. 1, 27-32 (1999).

48. Wang, L.-H., Südhof, T. C. \& Anderson, R. G. The appendage domain of $\alpha$-adaptin is high-affinity binding site for dynamin. J. Biol. Chem. 270, 10079-10083 (1995).

49. Micheva, K. D., Ramjaun, A. R., Kay, B. K. \& McPherson, P. S. SH3 domain-dependent interactions of endophilin with amphiphysin. FEBS Lett. 414, 308-312 (1997).

50. Zhou, W., Merrick, B. A., Khaledi, M. G. \& Tomer, K. B. Detection and sequencing of phosphopeptides affinity bound to immobilized metal ion beads by matrix-assisted laser desorption/ionization mass spectrometry. J. Am. Soc. Mass Spectrom. 11, 273-282 (2000). 


\section{Dynamin phosphorylation by Cdk5 stimulates GTPase activity}

The assay to measure the effect of phosphorylation on dynamin's GTPase activity initially involved a two step procedure: preincubation (to allow for dynamin phosphorylation) then a GTPase assay to measure its activity. First dynamin was phosphorylated by cdk5 or PKC in the presence of excess ATP or it was mock-phosphorylated in the presence of ATP alone, but no protein kinase. The concentration of dynamin phosphorylated was ten times the concentration to be used in the GTPase assay. Second, phosphorylated dynamin was immediately used for the GTPase assays after dilution ten-fold. This two-step procedure resulted in major artefacts in the results due to effects of the preincubation (phosphorylation) conditions themselves on dynamin GTPase activity, rather than as consequences resulting from phosphorylation. The effect of these artefacts were explored in great detail and only the major effects are discussed herein. The origin of these artefacts can be illustrated by a examples of conditions known to regulate dynamin GTPase activity. These include dynamin's capability to self-assemble into rings and helices that exhibit stimulated GTPase activity ${ }^{1-3}$. The selfassembly of dynamin is a cooperative process dependent upon the concentration of dynamin used ${ }^{4-9}$. The ionic strength of the buffers used in the GTPase assay can affect GTPase activity, since high $\mathrm{NaCl}$ inhibits dynamin's GTPase activity by preventing self-assembly ${ }^{2}$. The amount of calcium in the assay also affects GTPase activity. Calcium is required for PKC activation but inhibits dynamin ${ }^{10-12}$. Phospholipids such as PS and PtdIns $(4,5) \mathrm{P}_{2}$ are required to stimulate PKC but also stimulate dynamin ${ }^{4,13-15}$. Lastly, nucleotides such as ATP and GTP can regulate dynamin binding to lipids, microtubules and self-assembly ${ }^{10,11,16}$ and stimulate dynamin. Yet in order to phosphorylate dynamin prior to measuring its GTPase activity it was necessary to simultaneously subject it to all of these conditions. Ultimately the technical problem was solved by introducing a third step to the procedure: a salt disruption step.

Using the initial two-step procedure, both cdk5 and PKC $\alpha$ increased dynamin's GTPase activity. Dynamin was first phosphorylated by cdk 5 or PKC in vitro by incubating it with recombinant $\mathrm{PKC} \alpha$ or recombinant $\mathrm{cdk} 5 / \mathrm{p} 25$ for 1 hour at $30^{\circ} \mathrm{C}$ in the presence of $1 \mathrm{mM}$ ATP, $200 \mu \mathrm{M} \mathrm{Ca}^{2+}$ and $10 \mu \mathrm{g}$ PS. These conditions were previously known to result in stoichiometric phosphorylation of dynamin by PKC ${ }^{10}$. After phosphorylation reactions the GTPase activity of dynamin was measured. Following phosphorylation by PKC, dynamin's GTPase activity increased two-fold (fig S1a). This is consistent with previous reports of phosphorylation by PKC ${ }^{17}$. Following cdk5 phosphorylation, dynamin's GTPase activity was stimulated 3-fold (fig S1a).

Dynamin I can self-assemble into rings when dialysed from a solution containing high salt $(0.25$ to $1 \mathrm{M})$ to one containing low salt $(<20 \mathrm{mM})^{1}$. Dynamin was incubated for 1 hour at $30^{\circ} \mathrm{C}$ for the duration of the phosphorylation reaction under low salt conditions prior to being subjected to the GTPase assay. Thus there was a possibility that this preincubation period had allowed for the assembly of monomeric dynamin into higher order structures which then resulted in an artefactual stimulation of GTPase activity. The first set of control experiments involved incubating purified dynamin I, diluted with $20 \mathrm{mM}$ Tris (phosphorylation buffer) to concentrations similar to those used in the preincubation, for 1 hour at $30^{\circ} \mathrm{C}$. The GTPase activity of the preincubated dynamin was then measured in the presence and absence of $10 \mu \mathrm{g}$ PS (fig S1b). Purified dynamin I which was not subjected to the preincubation showed low GTPase activity. In the presence of PS, the GTPase activity was stimulated six-fold to maximal activity. Dynamin I which had been preincubated for 1 hour showed a three-fold increase in GTPase activity. Significantly, the addition of PS did not elicit any further increase in GTPase activity (fig S1b). Therefore preincubating dynamin for 1 hour resulted in the artefactual stimulation of basal GTPase activity which was no longer responsive to further stimulation by PS.
Next we aimed to determine if any one of the individual components of the phosphorylation reaction used in the preincubation had an effect on dynamin GTPase activity. Purified dynamin I was preincubated with ATP alone, PS alone or a combination of ATP and PS for 1 hour at $30^{\circ} \mathrm{C}$ before being subjected to a GTPase assay. As above, preincubation of dynamin alone resulted in a partial stimulation of its GTPase activity compared to dynamin which had not been preincubated (fig S1c). Furthermore, preincubated dynamin was no longer sensitive to further stimulation by PS (fig S1c, bars 3-4). Dynamin preincubated with ATP showed a similar result (bars 5-6). However, dynamin preincubated with PS revealed a complete inhibition of basal and PS-stimulated activity (bar 7-8). This major inhibitory effect of preincubation with PS was not seen when dynamin was incubated with PS and ATP together (bars 9-10), implying that the presence of ATP overcomes the effect of PS on dynamin. However, the addition of more PS during the GTPase assay still did not activate GTPase activity to maximal levels (bar 10).

These experiments indicate that the results shown in fig S1a are not interpretable. One possibility was that monomeric dynamin may have assembled into artefactual higher order/intermediate structures during the preincubation period. To test this, preincubated dynamin was subjected to SDS-PAGE using a 10\% non-denaturing gel. Following preincubation a number of higher molecular weight bands $(400 \mathrm{kDa})$ were formed which correspond to oligomers of dynamin (not shown). No oligomers were detected when $\beta$-mercaptoethanol was added, suggesting the formation of disulphide bonds (not shown).

Since spontaneously assembled dynamin rings can be dissociated in the presence of a modest level of salt $(>100 \mathrm{mM})^{1}$, the assembly of dynamin into higher order structures during the preincubation period might be prevented or reversed by the presence of salt $(\mathrm{NaCl})$. Prior to use in the preincubation reaction, it was necessary to determine if $\mathrm{NaCl}$ had any effect on dynamin I phosphorylation. However, we found that $150 \mathrm{mM} \mathrm{NaCl}$ completely inhibited both cdk5 and PKC phosphorylation of dynamin (not shown). Therefore $\mathrm{NaCl}$ could not be introduced during the preincubation (phosphorylation) reaction itself. Instead $\mathrm{NaCl}$ could be introduced after the preincubation and prior to the GTPase assay. Hence the assay was modified to comprise of three steps: preincubation (no salt present; 60 minutes at $30^{\circ} \mathrm{C}$ ) to allow for dynamin phosphorylation; a salt disruption step $(150 \mathrm{mM} \mathrm{NaCl}$ present; $10 \mathrm{~min}$ utes at $4^{\circ} \mathrm{C}$ ) to dissociate any higher order structures of dynamin formed during the preincubation, and the GTPase assay $(16 \mathrm{mM}$ $\mathrm{NaCl}$ present; 10 minutes at $37^{\circ} \mathrm{C}$ ). The experiment was designed such that dynamin was phosphorylated at a ten-fold higher concentration. Therefore when it was used in the final step (GTPase assay), the amount of $\mathrm{NaCl}$ was diluted to $15 \mathrm{mM}$ to ensure the final concentration of $\mathrm{NaCl}$ in the GTPase assay remained within the range tolerated by the GTPase assay $([\mathrm{NaCl}]>30 \mathrm{mM}$ inhibits dynamin's GTPase activity) ${ }^{11}$.

The three-step procedure was employed to address the effect of preincubation (phosphorylation) on subsequent dynamin GTPase activity. Purified dynamin I was preincubated with ATP alone, PS alone or ATP and PS for 1 hour at $30^{\circ} \mathrm{C}$. Following preincubation, the tubes were incubated on ice for 5 minutes before salt was added to $150 \mathrm{mM}$. The tubes were stored on ice for 10 minutes before being subjected to the GTPase assay (fig S1d). Following the addition of $\mathrm{NaCl}$, preincubated dynamin no longer showed a significant basal stimulation in GTPase activity. Furthermore, the addition of PS in the GTPase assay stimulated GTPase activity to maximal levels. Similar results were also observed for dynamin preincubated with ATP alone, PS alone or ATP and PS. All preincubation conditions tested now displayed sensitivity to PS stimulation. These results show that the addition of $\mathrm{NaCl}$ after the preincubation step and prior to the GTPase assay, was successful in restoring dynamin GTPase activity back to maximal levels.

The experiment now comprised of the preincubation (phosphorylation of dynamin), the salt disruption step and then the 
GTPase assay. Now the question of the effect of phosphorylation on dynamin GTPase activity was revisited using the new procedure. Purified dynamin I was incubated with purified recombinant GST$\mathrm{cdk} 5 / \mathrm{p} 25$ or PKC $\alpha$ in the presence of $1 \mathrm{mM}$ ATP, $10 \mu \mathrm{g}$ PS and 200 $\mu \mathrm{M} \mathrm{Ca}{ }^{2+}$ at $30^{\circ} \mathrm{C}$ for 1 hour. The phosphorylation reaction was stopped by storing the tubes on ice and $\mathrm{NaCl}$ was added to $150 \mathrm{mM}$ for 10 minutes on ice. The samples were then subjected to a standard GTPase assay. Dynamin phosphorylated by cdk5 showed a three-fold stimulation in GTPase activity (fig $2 \mathrm{~b}$ in manuscript). However, dynamin phosphorylated by PKC did not show a significant stimulation in GTPase activity. We conclude that the previous report of stimulated dynamin GTPase activity due to PKC phosphorylation is incorrect as it did not take the above factors into consideration ${ }^{17}$, but that $\mathrm{cdk} 5$ phosphorylation does increase dynamin's GTPase activity.

Additional Materials: Lipofectamine 2000 and Optimem serum-free media were from Invitrogen (USA). Anti-synapsin antibody was obtained from Santa Cruz (USA). Secondary fluorophore-coupled antibodies were obtained from Jackson Immunoresearch Laboratories (West Haven, PA) and used according to manufacturer's instructions. Coverslips were from Raymond Lamb (Eastbourne, U.K.) Tissue culture plastics were from Falcon (Plymouth, U.K.) or Griener (Dursley, U.K.). Penicillin/streptomycin, phosphate buffered salts, foetal calf serum and Minimal Essential Medium were from Invitrogen (Paisley, Strathclyde, U.K.). Scintillation vials were from Canberra Packard (Pangbourne, Berks, U.K.). FM4-64 was from Molecular Probes (Oregon, USA).

GTPase assay: Dynamin GTPase activity was determined by hydrolysis of $\left[\gamma^{-32} \mathrm{P}\right]$ GTP by a method modified from that used previously ${ }^{17}$. Purified dynamin I $(0.2 \mu \mathrm{g} /$ tube $)$ was incubated in GTPase buffer ( $10 \mathrm{mM}$ Tris, $10 \mathrm{mM} \mathrm{NaCl}, 2 \mathrm{mM} \mathrm{Mg} \mathrm{m}^{2+}, 0.05 \%$ Tween $80, \mathrm{pH} 7.4,1 \mu \mathrm{g} / \mathrm{ml}$ leupeptin and 0.1 $\mathrm{mM}$ PMSF $)$ and a GTP cocktail containing $0.3 \mathrm{mM}$ GTP and $1.3 \mu \mathrm{Ci}\left[\gamma_{-}{ }^{32} \mathrm{P}\right]-\mathrm{GTP}$ in the presence or absence of kinase cofactors for $10 \mathrm{~min}$ at $30^{\circ} \mathrm{C}$. The final assay volume was $40 \mu$. Dynamin activity was measured as either basal or phospholipid-stimulated with the addition of $5 \mu \mathrm{g} / \mathrm{ml} \mathrm{L-phos-}$ phatidylserine. The reaction was terminated with $100 \mu$ of GTPase stop buffer (2\% formic acid, $8 \%$ acetic acid, $\mathrm{pH} 1.9$ ), followed by $600 \mu \mathrm{l}$ of acid-washed charcoal solution ( $7 \%$ charcoal in acidic solution $(\mathrm{w} / \mathrm{v}))$ and $100 \mu \mathrm{lBSA}(5 \mathrm{mg} / \mathrm{ml})$. After centrifuging for $5 \mathrm{~min}(13,000 \mathrm{rpm}$ at room temperature), $200 \mu$ l of each supernatant was counted in a $\beta$-counter for the release of ${ }^{32} \mathrm{P}_{\mathrm{i}}$ from $\left[\gamma^{32} \mathrm{P}\right]-\mathrm{GTP}$. Transfection of B104 neurons: Rat brain derived B104 neuroblastoma cells ${ }^{18,19}$ were seeded on poly-Dlysine coated glass coverslips in a 12-well plate at low density ( 2000 cells/well). Cells were incubated overnight with DMEM supplemented with $10 \% \mathrm{FCS}$ at $37^{\circ} \mathrm{C}$ in a $5 \% \mathrm{CO}_{2}$ humidified incubator. Media was replaced with $100 \mu \mathrm{M}$ dibutyryl-cAMP/1\% horse serum in DMEM to differentiate cells. After 7-10 days, cells were transiently transfected with EGFP-WT-cdk5 $(1 \mu \mathrm{g})$ or EGFP-DN-cdk5-T33 $(1 \mu \mathrm{g})$ using Lipofectamine $2000(1.5 \mu \mathrm{l})$ in Optimem serum-free media $(1 \mathrm{ml})$ according to the manufacturers' instructions. After 5 hours of transfection, media was replaced with $100 \mu \mathrm{M}$ dibutyrylcAMP $/ 1 \%$ horse serum in DMEM and cells were incubated for a further 1-3 days. Cells were then fixed in $4 \%$ paraformaldehyde for 20 min, permeabilized with $0.1 \%$ SDS in blocking buffer (1\% BSA in PBS) for $15 \mathrm{~min}$, and then incubated in blocking buffer for 1 hour at room temperature. Cells were incubated with primary antibodies at appropriate dilutions (synapsin, 1:200; phospho-dynamin, 1: 3000; dynamin, 1:2000) in blocking buffer overnight at $4{ }^{\circ} \mathrm{C}$, and washed extensively with PBS before incubating with secondary Texas Red-coupled antibodies (1:200 in PBS) for $1 \mathrm{hr}$ at room temperature Coverslips were mounted on glass slides and images were acquired with a Leica DMLB fluorescence microscope equipped with appropriate filter sets and a cooled CCD camera (SPOT2; Diagnostic Instruments, Sterling Heights, MI). Images were further processed for illustrative purposes using Adobe Photoshop 6.0.

Cerebellar granule neurons: Cerebellar granule neuron (CGN) cultures were prepared from the cerebella of 7-day old Sprague-Drawley rat pups essentially as previously described ${ }^{20}$. Neurons were plated on poly-D-lysine coated glass coverslips at a density of $0.25 \times 10^{6}$ cells/coverslip for single cell imaging and $0.75 \times 10^{6}$ for transmitter release studies. Neurons were cultured in Minimal Essential Medium (MEM) containing Earle's salts (GIBCO) plus 10\% (v/v) foetal calf serum, $25 \mathrm{mM} \mathrm{KCl}, 30 \mathrm{mM}$ glucose, $2 \mathrm{mM}$ glutamine, $100 \mathrm{U} / \mathrm{ml}$ penicillin and $100 \mu \mathrm{g} / \mathrm{ml}$ streptomycin at $37^{\circ} \mathrm{C}$, in a humidified atmosphere of $5 \% \mathrm{CO}_{2}: 95 \%$ air. Culture medium was supplemented with $10 \mu \mathrm{M}$ cytosine arabinoside after 24 hours in vitro. Neurons were used between 9 - 11 days in vitro in all experiments. Transfection of cerebellar granule neurons: CGNs were co-transfected with GFP and either WT-Cdk5 or DN-Cdk $5^{21}$ using an established calcium phosphate precipitation protocol ${ }^{22}$. CGNs were transiently transfected at 7 days in vitro and were routinely used 48 hours later.

Synaptic vesicle recycling in cerebellar granule neurons: The effect of overexpression of either WTCdk5 or DN-Cdk5 on synaptic vesicle recycling in CGNs was monitored using the styryl dye FM4-64 ${ }^{23}$. Neurons were removed from culture medium and left to repolarise for $10 \mathrm{~min}$ in incubation medium (170 mM NaCl, $3.5 \mathrm{mM} \mathrm{KCl}, 0.4 \mathrm{mM} \mathrm{KH}_{2} \mathrm{PO}_{4}, 20 \mathrm{mM}$ TES ( $N$-tris[hydroxy-methyl]-methyl-2aminoethane-sulphonic acid), $5 \mathrm{mM} \mathrm{NaHCO}, 5$ mM glucose, $1.2 \mathrm{mM} \mathrm{Na}_{2} \mathrm{SO}_{4}, 1.2 \mathrm{mM} \mathrm{MgCl}_{2}, 1.3$ $\mathrm{mM} \mathrm{CaCl}_{2}, \mathrm{pH}$ 7.4). Neurons were then stimulated for $10 \mathrm{~s}$ using $\mathrm{KCl}$ stimulation medium (incubation medium supplemented with $50 \mathrm{mM} \mathrm{KCl}, 50 \mathrm{mM} \mathrm{NaCl}$ being removed to maintain osmolarity) and left to repolarise for $7 \mathrm{~min}$. CGNs were then loaded with FM4-64 by evoking SV recycling using $\mathrm{KCl}$ stimulation medium (supplemented with $10 \mu \mathrm{M}$ FM4-64) for $2 \mathrm{~min}$. Neurons were then returned to incubation medium supplemented with $10 \mu \mathrm{M}$ FM4-64 for one min to allow completion of endocytosis $^{24}$. Neurons were washed with incubation medium and mounted on an epifluorescence microscope (Zeiss Axiovert TV-100). After 15 min FM4-64 was unloaded from the cells by perfusing KCl stimulation medium for $30 \mathrm{sec}$. FM4-64 unloading was visualised using a Zeiss Plan-Apochromat x63 / 1.4 NA oil immersion objective at $550 \mathrm{~nm}$ excitation and a band-pass emission filter of 600-640 nm. GFP in transfected CGNs were visualised using $480 \mathrm{~nm}$ excitation and a band-pass emission filter of 510$550 \mathrm{~nm}$. The decrease in fluorescence intensity on stimulated FM4-64 unloading from nerve terminals was visualised with a Hammamatsu Orca-ER CCD digital camera (Hammamatsu City, Japan) and offline imaging software (Compix Imaging Systems, USA). The extent of FM4-64 loading was quantified by monitoring the total decrease in fluorescence on unloading with the $\mathrm{KCl}$ stimulus. The kinetics of FM4-64 unloading were estimated by normalising the start and completion of unloading to 1 and 0 respectively.

Lipid binding assay: The effect of cdk5 phosphorylation on dynamin's ability to bind phospholipids was examined using controlled pore glass beads coated with phosphatidylserine ${ }^{10,25}$. Purified dynamin I was incubated with purified recombinant PKC $\alpha$ or recombinant GST-Cdk5/p25 in the presence of $100 \mu \mathrm{M}$ ATP, $10 \mu \mathrm{g}$ PS and $200 \mu \mathrm{M} \mathrm{Ca}^{2+}$ for 1 hour at $30^{\circ} \mathrm{C}$. The resulting phosphorylation mix was passed through a Sephadex G-10 column to remove the salt and the ATP which have been shown previously to affect dynamin's interaction with PS ${ }^{10}$. PS-coated glass beads were added to each of the samples, incubated on ice for 20 minutes and supernatants and pellets were separated and run on gels. Initial experiments were unsuccessful as the unphosphorylated dynamin was frequently proteolysed. The protease activity was found to derive from the phospholipid solution used to coat the beads. To eliminate dynamin degradation, the lipid-coated beads were treated with $10 \mu \mathrm{g} / \mathrm{ml}$ leupeptin and $1 \mathrm{mM}$ of PMSF for 1 hour on ice prior to use in the lipid-binding assay.

1. Hinshaw, J. E. \& Schmid, S. L. Dynamin self-assembles into rings suggesting a mechanism for coated vesicle budding. Nature 374, 190-192 (1995).

2. Warnock, D. E., Hinshaw, J. E., \& Schmid, S. L. Dynamin self-assembly stimulates its GTPase activity. J.Biol.Chem. 271, 22310-22314 (1996).

3. Muhlberg, A. B., Warnock, D. E., \& Schmid, S. L. Domain structure and intramolecular regulation of dynamin GTPase. EMBO J. 16, 6676-6683 (1997).

4. Tuma, P. L. \& Collins, C. A. Activation of dynamin GTPase is a result of positive cooperativity. J.Biol.Chem. 269, 30842-30847 (1994).

5. Tuma, P. L. \& Collins, C. A. Dynamin forms polymeric complexes in the presence of lipid vesicles. Characterization of chemically cross-linked dynamin molecules.

J.Biol.Chem. 270, 26707-26714 (1995).

6. Zalewski, P. D., Forbes, I. J., Valente, L., Apostolou, S., \& Hurst, N. P. Translocation of protein kinase $\mathrm{C}$ to a Triton-insoluble sub- cellular compartment induced by the lipophilic gold compound auranofin. Biochem.Pharmacol. 37, 1415-1417 (1988).

7. Stowell, M. H., Marks, B., Wigge, P., \& McMahon, H. T. Nucleotide-dependent conformational changes in dynamin: evidence for a mechanochemical molecular spring. Nat.Cell Biol. 1, 27-32 (1999).

8. Binns, D. D. et al. Correlation between self-association modes and GTPase activation of dynamin. J.Protein Chem. 18, 277-290 (1999).

9. Hinshaw, J. E. Dynamin and its role in membrane fission. Annu.Rev.Cell Dev.Biol. 16, 483-519 (2000).

10. Liu, J. P., Powell, K. A., S dhof, T. C., \& Robinson, P. J. Dynamin I is a $\mathrm{Ca}^{2+}$-sensitive phospholipid-binding protein with very high affinity for protein kinase C. J.Biol.Chem. 269, 21043-21050 (1994)

11. Liu, J. P., Zhang, Q.-X., Baldwin, G., \& Robinson, P. J. Calcium binds dynamin I and inhibits its GTPase activity. J.Neurochem. 66, 2074-2081 (1996).

12. Cousin, M. A. \& Robinson, P. J. $\mathrm{Ca}^{2+}$ inhibition of dynamin arrests synaptic vesicle recycling at the active zone. J.Neurosci. 20, 949-957 (2000).

13. Tuma, P. L., Stachniak, M. C., \& Collins, C. A. Activation of dynamin GTPase by acidic phospholipids and endogenous rat brain vesicles. J.Biol.Chem. 268, 17240-17246 (1993). 14. Zheng, J. et al. Identification of the binding site for acidic phospholipids on the $\mathrm{PH}$ domains of dynamin: implications for stimulation of GTPase activity. J.Mol.Biol. 255, 1421 (1996).

15. Lin, H. C., Barylko, B., Achiriloaie, M., \& Albanesi, J. P. Phosphatidylinositol (4,5)bisphosphate-dependent activation of dynamins I and II lacking the Proline/Arginine-rich domains. J.Biol.Chem. 272, 25999-26004 (1997).

16. Maeda, K., Nakata, T., Noda, Y., Sato-Yoshitake, R., \& Hirokawa, N. Interaction of dynamin with microtubules: its structure and GTPase activity investigated by using highly purified dynamin. Mol.Biol.Cell 3, 1181-1194 (1992).

17. Robinson, P. J. et al. Dynamin GTPase regulated by protein kinase C phosphorylation in nerve terminals. Nature 365, 163-166 (1993).

18. Schubert, D. et al. Clonal cell lines from the rat central nervous system. Nature 249, 224-227 (1974).

19. Gu, X. Q. \& Waxman, S. G. Action potential-like responses in B104 cells with low $\mathrm{Na}+$ channel densities. Brain Res. 735, 50-58 (1996).

20. Cousin, M. A., Pocock, J. M., \& Nicholls, D. G. Intracellular free Ca2+ responses in electrically stimulated cerebellar granule cells. Biochem.Soc.Trans. 23, 648-652 (1995). 21. van den Heuvel, S. \& Harlow, E. Distinct roles for cyclin-dependent kinases in cell cycle control. Science 262, 2050-2054 (1993).

22. Koulich, E., Nguyen, T., Johnson, K., Giardina, C., \& D mello, S. NF-kappaB is involved in the survival of cerebellar granule neurons: association of IkappaBbeta phosphorylation with cell survival. J.Neurochem. 76, 1188-1198 (2001).

23. Cousin, M. A. \& Nicholls, D. G. Synaptic vesicle recycling in cultured cerebellar granule cells: Role of vesicular acidification and refilling. J.Neurochem. 69, 1927-1935 (1997). 24. Ryan, T. A. et al. The kinetics of synaptic vesicle recycling measured at single presynaptic boutons. Neuron 11, 713-724 (1993).

25. Ando, Y. et al. Enhancement of calcium sensitivity of lipocortin I in phospholipid binding induced by limited proteolysis and phosphorylation at the amino terminus as analyzed by phospholipid affinity column chromatography. J.Biol.Chem. 264, 6948-6955 (1989). 

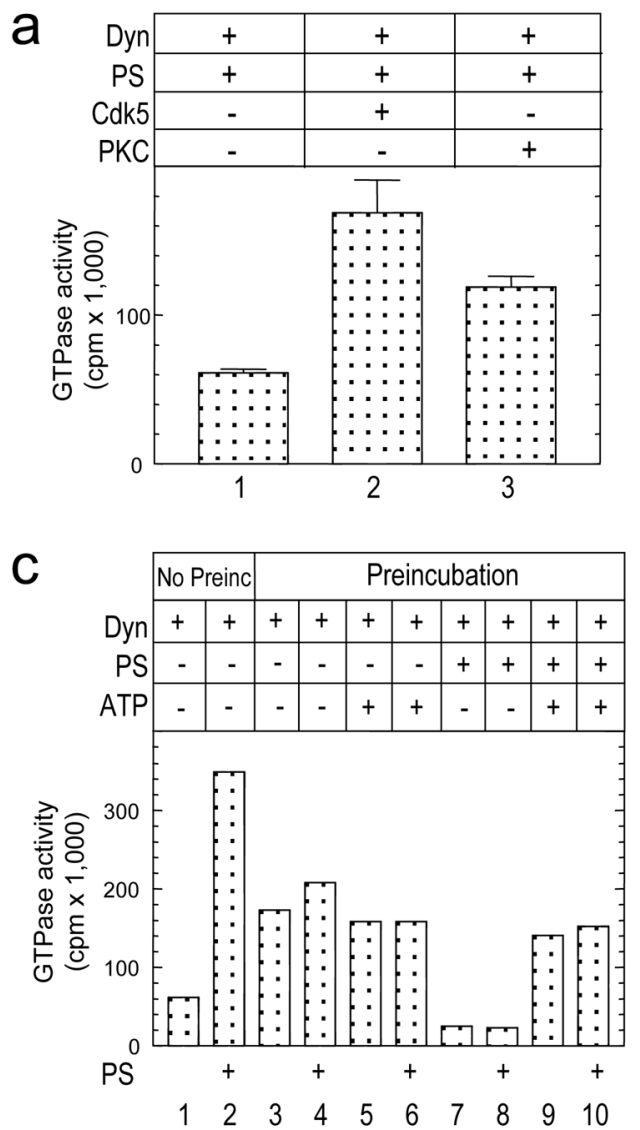

Fig S1a Effect of cdk5 phosphorylation on dynamin's GTPase activity. a. The effect of dynamin phosphorylation in the initial two-stage GTPase assay. Purified dynamin I ( $5 \mu \mathrm{g}$ ) was phosphorylated with recombinant GST-cdk5/p25 or PKC in the presence of $1 \mathrm{mM} \mathrm{Mg} / \mathrm{ATP}, 10 \mu \mathrm{g} / \mathrm{ml} \mathrm{PS}$ and $200 \mu \mathrm{M} \mathrm{Ca}{ }^{2+}$ at $30^{\circ} \mathrm{C}$ for 1 hour. Phosphorylated dynamin I was then subjected to a GTPase assay. The labels above the figure indicate the factors present during the preincubation step. b. The effect of preincubation with Mg/ATP on dynamin I GTPase activity. Purified dynamin I ( $5 \mu \mathrm{g}$ ) was preincubated with Mg/ATP for 1 hour at $30^{\circ} \mathrm{C}$ (no protein kinase) and subjected to a GTPase assay \pm PS $(10 \mu \mathrm{g} / \mathrm{mll})$. Preincubation elevated basal activity and abolished PS-stimulated activity. c. The effects of preincubating dynamin with PS, Mg/ATP or PS + ATP on GTPase activity. Dynamin was preincubated as in (b) with PS $(10 \mu \mathrm{g} / \mathrm{mll})$, ATP (1 mM) or PS plus ATP for 1 hour at $30^{\circ} \mathrm{C}$
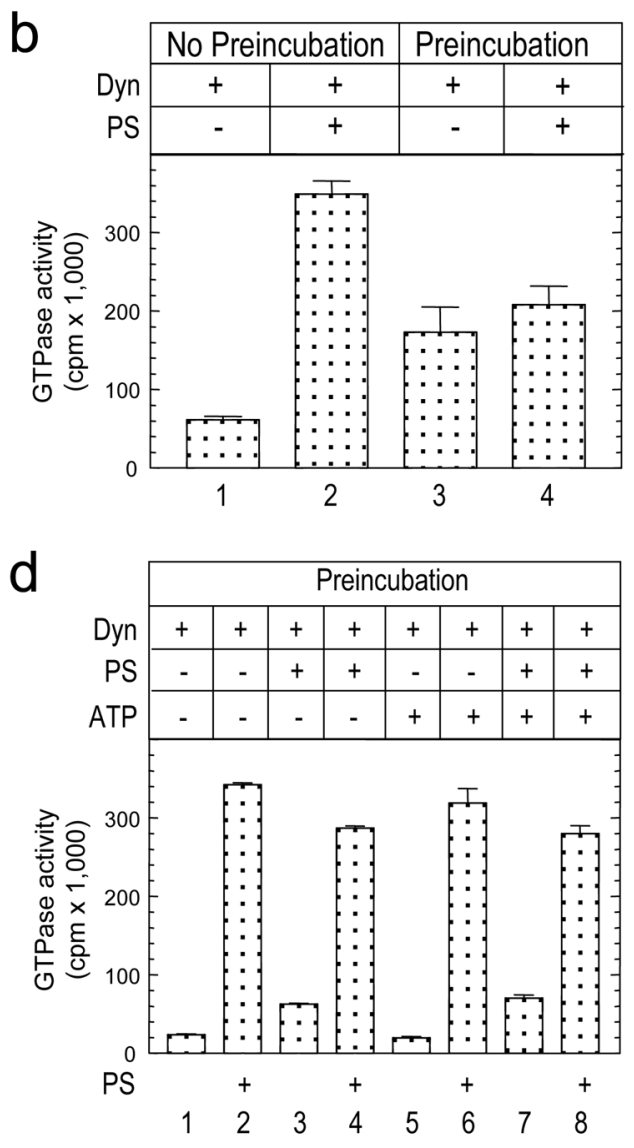

and subjected to a GTPase assay \pm PS $(10 \mu \mathrm{g})$. Note that PS was added twice, during the preincubation (indicated above the panel) and again as a stimulus for the GTPase assay (below the panel). All preincubation conditions abolished the PSdependence of the GTPase assay. $\mathbf{d}$. The three-stage GTPase assay introduces a salt disruption step. Purified dynamin I (5 $\mu \mathrm{g})$ was preincubated in the absence and presence of PS $(10 \mu \mathrm{g} / \mathrm{ml})$ ATP $(1 \mathrm{mM})$ or both for 1 hour at $30^{\circ} \mathrm{C}$ (indicated above the panel). After preincubation, $\mathrm{NaCl}$ was added to $150 \mathrm{mM}$ for 10 minutes on ice (salt disruption step) and then samples were subjected to a GTPase assay in the presence or absence of PS (10 $\mu \mathrm{g} / \mathrm{ml}$, indicated below the panel). The salt disruption step restored the PS-dependence of the GTPase assay. All results are representative of at least two independent experiments, each performed in triplicate. 


\section{supplementary infomation}

a

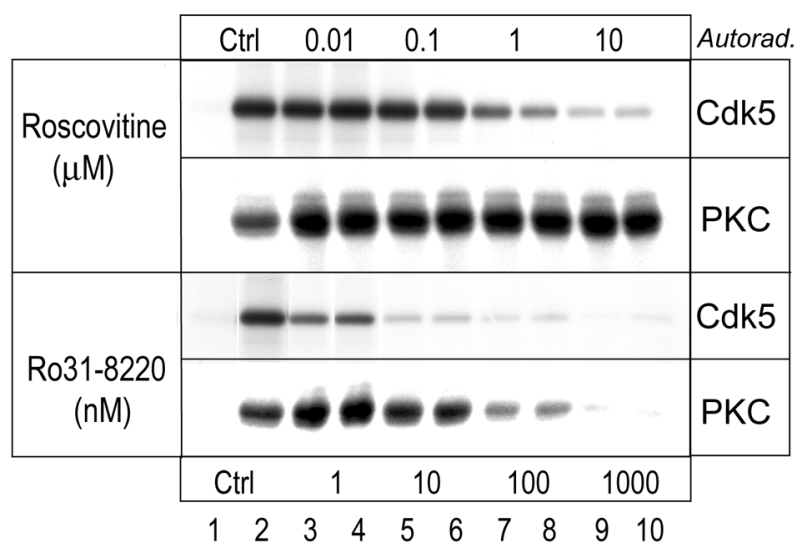

b

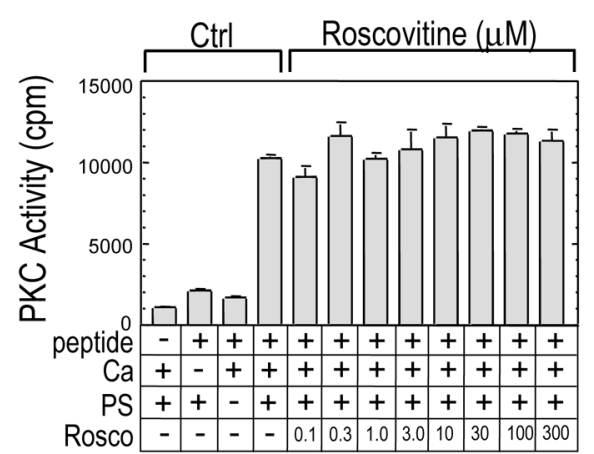

Fig S2 Roscovitine inhibits dynamin I phosphorylation by cdk5 but not by PKC- $\alpha$ in vitro. a. Dynamin I was phosphorylated in vitro by cdk5 or PKC- $\alpha$ in the absence (lane 2) or presence (lanes 3-10, each drug was employed in duplicate) of increasing concentrations of roscovitine or Ro31-8220. Basal phosphorylation without protein kinase is shown in lane 1 of the autoradiograph. b. Lack of effect of an extended concentration range of roscovitine on PKC activity in a standard PKC assay using a synthetic peptide substrate. Basal kinase activity in the absence of peptide, $\mathrm{Ca}^{2+}$ or phosphatidylserine (PS) are shown in the first 3 bars and maximal PKC activity in the presence of the activators $\mathrm{Ca}^{2+}$ and PS is shown in the 4th. Results are the mean \pm SEM for $n=3$.

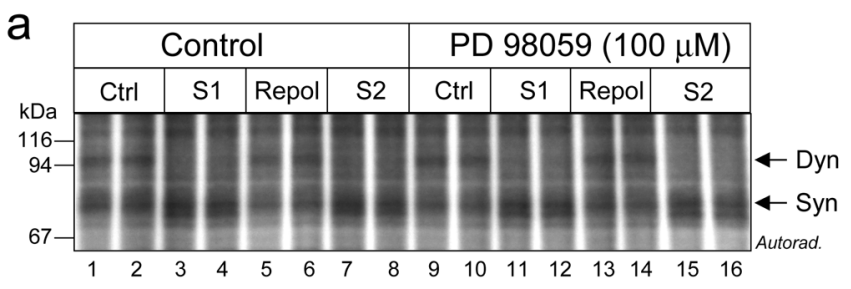

b

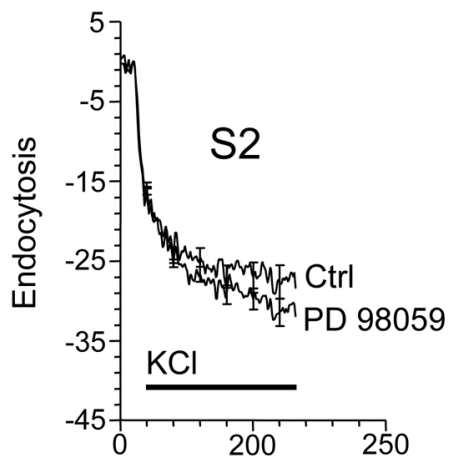

Fig S3 Lack of effect of PD 98059 on dynamin I phosphorylation and SVE. a. P2 synaptosomes were prepared from rat brain, labelled with ${ }^{32} \mathrm{P}_{\mathrm{i}}$ and lysed either before stimulation (Ctrl), after stimulation with $41 \mathrm{mM} \mathrm{KCl}$ for $10 \mathrm{~s}$ (S1), 7 min after repolarisation (Repol) or 10 s after as second stimulation with $41 \mathrm{mM} \mathrm{KCl}$ (S2). Synaptosomes were preincubated for $15 \mathrm{~min}$ in the absence or presence of $100 \mu \mathrm{M}$ PD 98059. Note that the drug was included in all subsequent buffers as well as the preincubation. Samples were separated by SDS-PAGE and an autoradiograph is presented. Arrows indicate either dynamin I (Dyn) or synapsin I (Syn, doublet). Results are in duplicate and are representative of at least three experiments. b. Endocytosis at S2 is not blocked by PD 98059. Synaptosomes were preincubated with $100 \mu \mathrm{M}$ PD 9805915 minutes before stimulation with $30 \mathrm{mM} \mathrm{KCl}$ in the presence of $\mathrm{Ca}^{2+}$. Synaptosomes were repolarised and loaded with FM2-10 (S2) by stimulation with $30 \mathrm{mM} \mathrm{KCl}$ either in the presence or absence of $\mathrm{Ca}^{2+}$.

Synaptosomes were continually incubated with PD 98059 for all stages up to and including this point. The subsequent release of loaded FM2-10 by a standard stimulus of $30 \mathrm{mM} \mathrm{KCl}$ in the presence of $\mathrm{Ca}^{2+}$ is displayed after subtraction of traces loaded in the absence of $\mathrm{Ca}^{2+}$. Traces are representative and normalised to an arbitrary value $(n=3)$. 

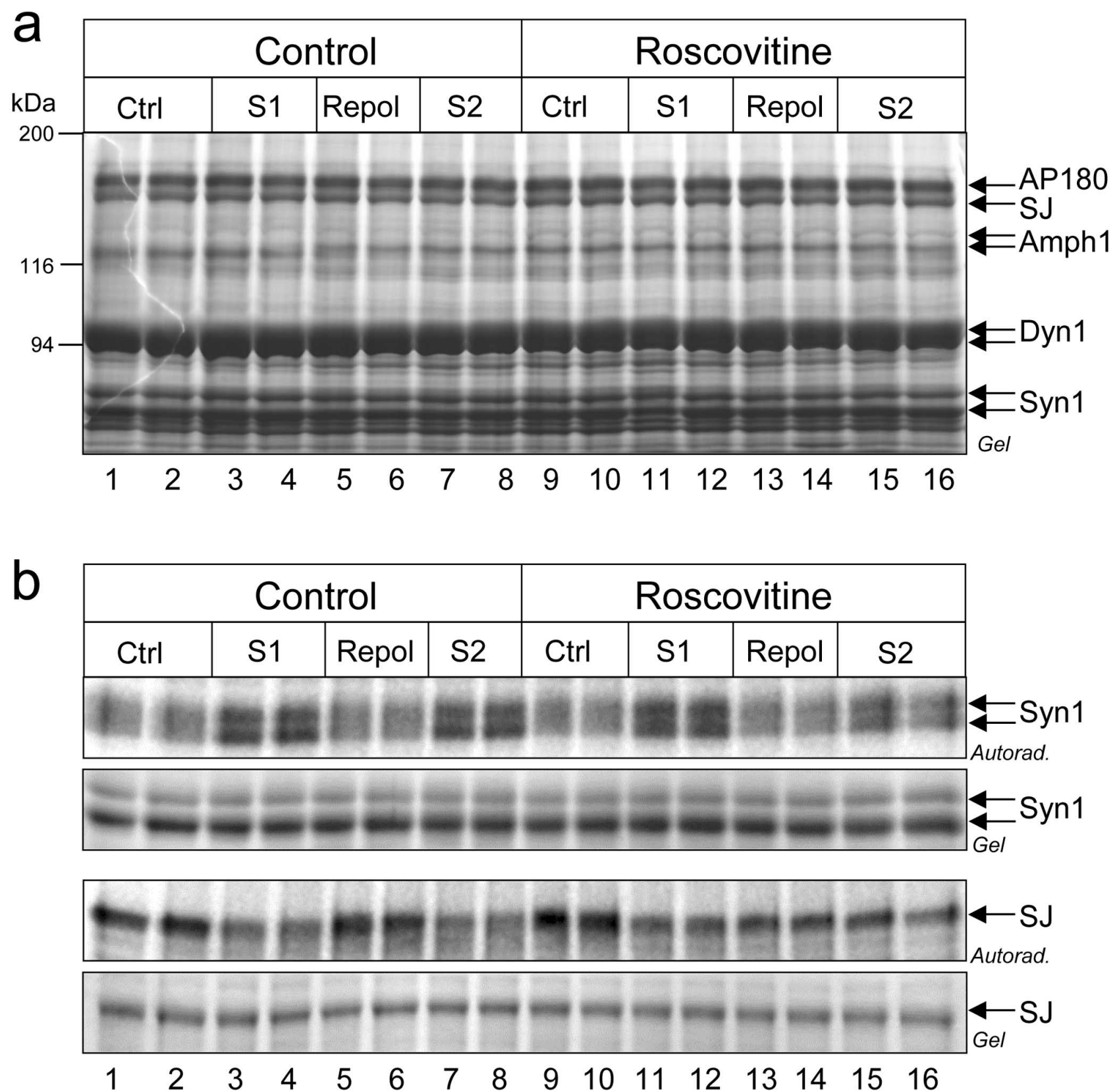

Fig S4 Double pull-down of 4 dephosphins and synapsin from synaptosomes. a. Equal protein load. A Coomassie blue stained gel is presented from a pull-down shown in the manuscript fig $4 \mathrm{e} .{ }^{32} \mathrm{P}_{\mathrm{i}}$-labelled synaptosomes were stimulated twice in the presence or absence of roscovitine as described in the legend to fig $4 \mathrm{e}$. The lysed synaptosomes were incubated with a mixture of GSH beads with recombinant GST- $\alpha$-adaptin appendage domain plus GST-endophilin 1-SH3 domain. Equal protein was extracted with each condition. Each protein indicated was identified by MALDITOF mass spectrometry as well as by western blot (not shown). b. Effect of roscovitine on synapsin I phosphorylation. The experiment was the same as in panel a, except only the regions of the gel containing synaptojanin (bottom two panes) or synapsin I (top two panes) is shown. The Coomassie stain shows that equal protein was recovered in all pull-downs. Roscovitine blocked synaptojanin rephosphorylation (lanes 13-14). However, the increase in synapsin phosphorylation at S1 was completely unaffected by the drug (lanes 11-12). Synapsin phosphorylation increase at S2 was partly reduced. The same results were obtained in 4 additional experiments. 


\section{supplementary infomation}
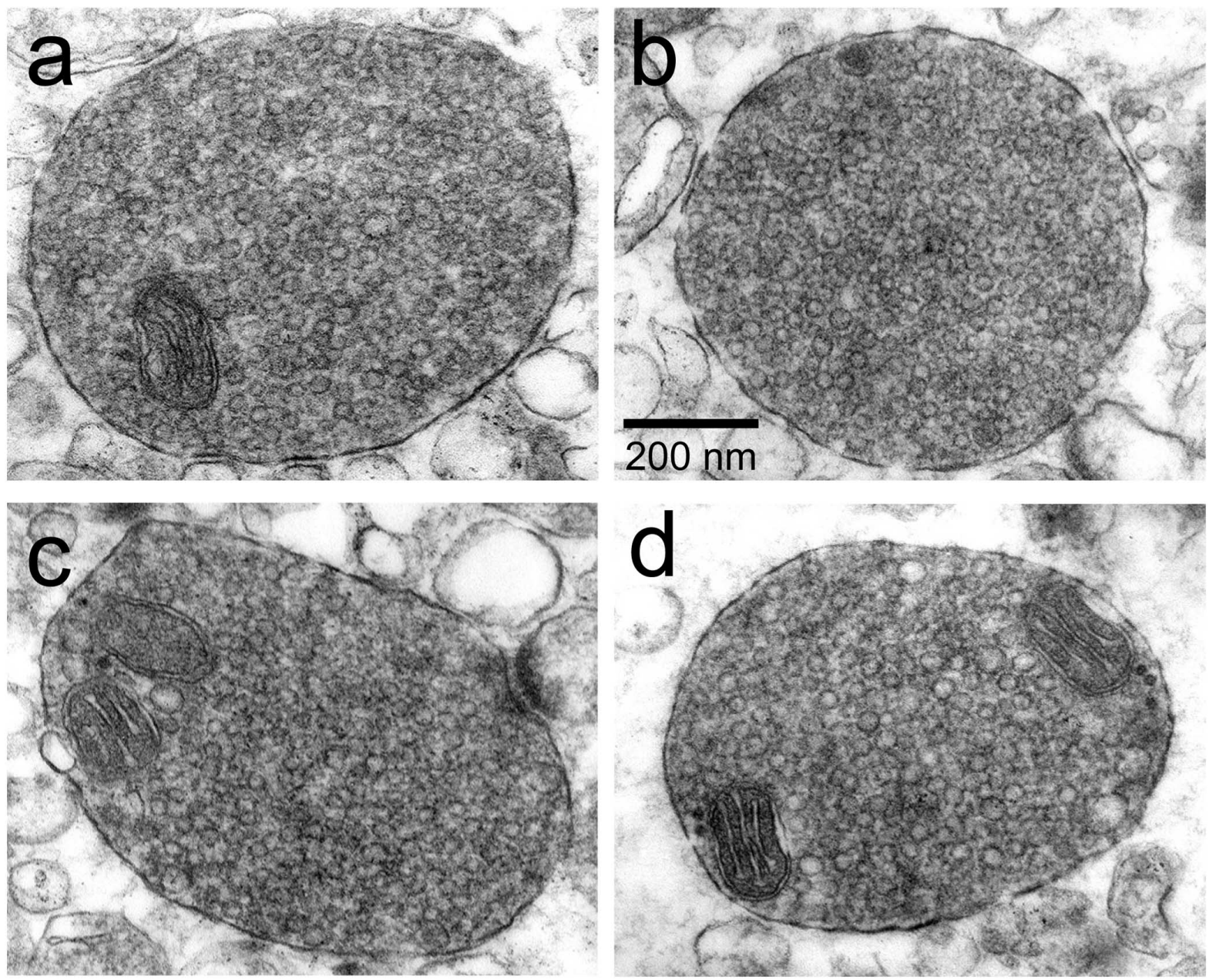

Fig S5 Morphology of synaptosomes at rest or after two cycles of stimulus-recovery. Percoll purified synaptosomes from rat brain were subjected to two cycles of stimulation with $41 \mathrm{mM} \mathrm{KCl}(10 \mathrm{~s})$ and aliqouts of synaptosomes at rest, S1, repolarised and S2 states were fixed in 5\% glutaraldehyde (in PBS pH 7.4) and processed for electron microscopy by conventional methods. The morphology at

rest (a), S1 (b), repolarised (c) and S2 (d) of representative synaptosomes are shown. Synaptosomes are filled with synaptic vesicles, contain 1 or more mitochondrial profile and have a smooth, sealed plasma membrane. The images are representative of at least 6 independent experiments. Scale bar $=200 \mathrm{~nm}$. 

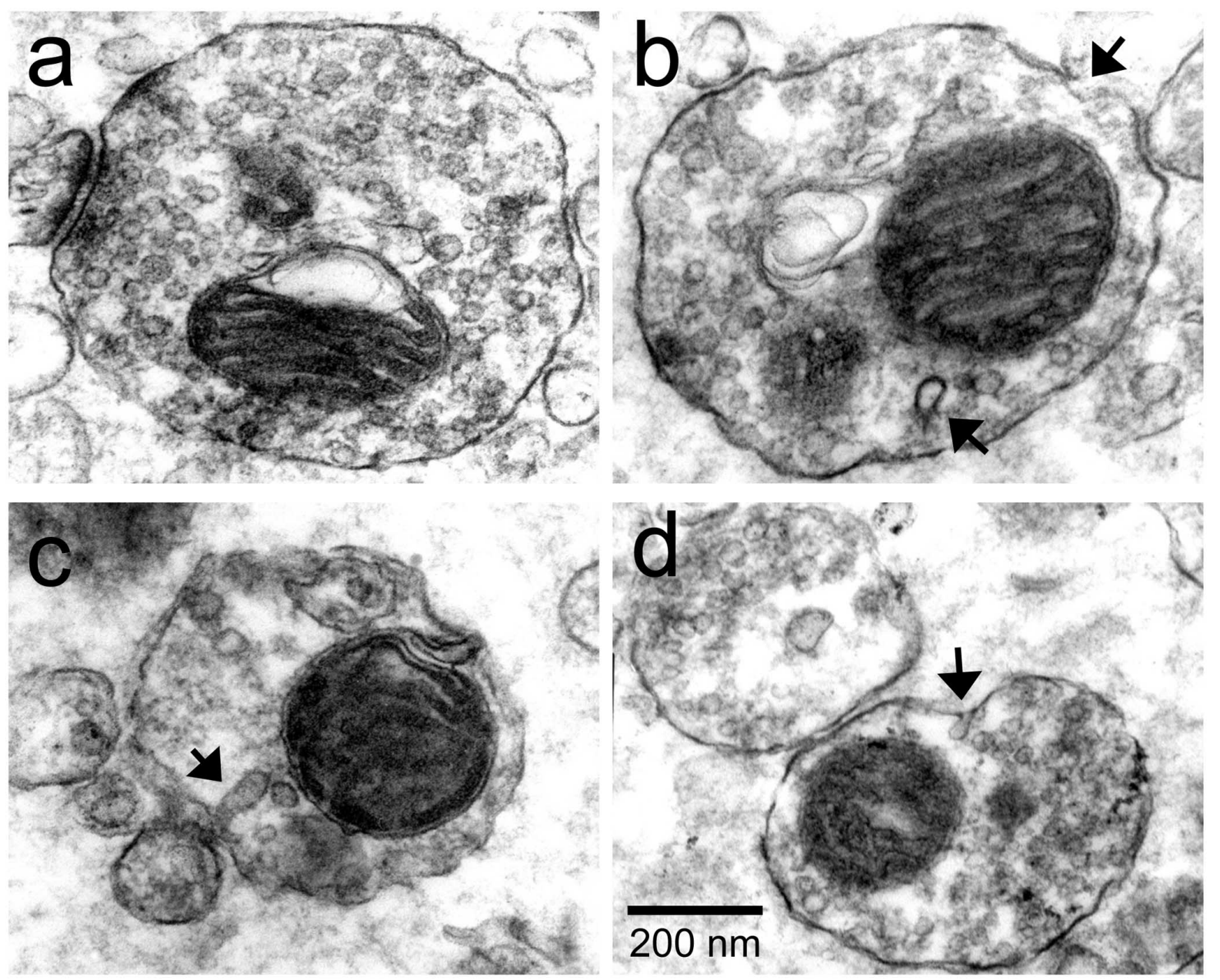

Fig S6 Morphology of synaptosomes during S2 in the presence of roscovitine. A gallery of electron micrographs of synaptosomes treated with roscovitine at S2. The majority of synaptosomes were completely empty of SVs, therefore they could not be proven to actually be synaptosomes by the main morphological criteria. A smaller number of synaptosomes (as shown in this gallery,

a-d) varying in size are representative of the effect of roscovitine on all other synaptosomes at S2. All these synaptosomes display mitochondrial profiles, membrane ruffling and vesicle depletion as observed in fig 7c. Arrows highlight membrane invaginations and potential endocytic profiles indicative of an endocytic block. Scale bar $=200 \mathrm{~nm}$. 